\title{
Hankel operators induced by radial Bekollé-Bonami weights on Bergman spaces
}

\author{
José Ángel Peláez ${ }^{1} \cdot$ Antti Perälä ${ }^{2}$ Jouni Rättyä ${ }^{3}$
}

Received: 26 June 2018 / Accepted: 4 October 2019 / Published online: 7 November 2019

(c) The Author(s) 2019

\begin{abstract}
We study big Hankel operators $H_{f}^{v}: A_{\omega}^{p} \rightarrow L_{v}^{q}$ generated by radial Bekollé-Bonami weights $v$, when $1<p \leq q<\infty$. Here the radial weight $\omega$ is assumed to satisfy a two-sided doubling condition, and $A_{\omega}^{p}$ denotes the corresponding weighted Bergman space. A characterization for simultaneous boundedness of $H_{f}^{v}$ and $H_{\frac{v}{f}}$ is provided in terms of a general weighted mean oscillation. Compared to the case of standard weights that was recently obtained by Pau et al. (Indiana Univ Math J 65(5):1639-1673, 2016), the respective spaces depend on the weights $\omega$ and $v$ in an essentially stronger sense. This makes our analysis deviate from the blueprint of this more classical setting. As a consequence of our main result, we also study the case of anti-analytic symbols.
\end{abstract}

Keywords Hankel operator · Bekollé-Bonami weight · Bergman space · Bergman projection $\cdot$ doubling weight

Mathematics Subject Classification Primary 47B35; Secondary 32A36

This research was supported in part by Ministerio de Economía y Competitivivad, Spain, projects MTM2014-52865-P and MTM2015-69323-REDT; La Junta de Andalucía, project FQM210; Academy of Finland Project No. 268009. AP acknowledges financial support from the Spanish Ministry of Economy and Competitiveness, through the María de Maeztu Programme for Units of Excellence in R\&D

(MDM-2014-0445), Academy of Finland Project No. 268009, and the Grant MTM2017-83499-P

(Ministerio de Educación y Ciencia).

Antti Perälä

antti.perala@gu.se

José Ángel Peláez

japelaez@uma.es

Jouni Rättyä

jouni.rattya@uef.fi

1 Departamento de Análisis Matemático, Universidad de Málaga, Campus de Teatinos, 29071 Málaga, Spain

2 Department of Mathematical Sciences, Chalmers University of Technology and the University of Gothenburg, 41296 Gothenburg, Sweden

3 Department of Physics and Mathematics, University of Eastern Finland, P.O.Box 111, 80101 Joensuu, Finland 


\section{Introduction and main results}

Let $\mathcal{H}(\mathbb{D})$ denote the space of analytic functions in the unit disc $\mathbb{D}=\{z \in \mathbb{C}:|z|<1\}$. A function $\omega: \mathbb{D} \rightarrow[0, \infty)$, integrable over the unit disc $\mathbb{D}$, is called a weight. It is radial if $\omega(z)=\omega(|z|)$ for all $z \in \mathbb{D}$. For $0<p<\infty$ and a weight $\omega$, the Lebesgue space $L_{\omega}^{p}$ consists of (equivalence classes of) complex-valued measurable functions $f$ in $\mathbb{D}$ such that

$$
\|f\|_{L_{\omega}^{p}}=\left(\int_{\mathbb{D}}|f(z)|^{p} \omega(z) d A(z)\right)^{\frac{1}{p}}<\infty,
$$

where $d A(z)=d x d y / \pi$ denotes the normalized Lebesgue area measure on $\mathbb{D}$. The weighted Bergman space $A_{\omega}^{p}$ is the space of analytic functions in $L_{\omega}^{p}$. As usual, $A_{\alpha}^{p}$ denotes the weighted Bergman space induced by the standard radial weight $(\alpha+1)\left(1-|z|^{2}\right)^{\alpha}$. If $v$ is a radial weight then $A_{v}^{2}$ is a closed subspace of $L_{v}^{2}$, and the orthogonal projection from $L_{v}^{2}$ to $A_{v}^{2}$ is given by

$$
P_{\nu}(f)(z)=\int_{\mathbb{D}} f(\zeta) \overline{B_{z}^{v}(\zeta)} v(\zeta) d A(\zeta), \quad z \in \mathbb{D},
$$

where $B_{z}^{v}$ are the reproducing kernels of $A_{v}^{2} ; f(z)=\left\langle f, B_{z}^{v}\right\rangle_{A_{v}^{2}}$ for all $z \in \mathbb{D}$ and $f \in A_{v}^{2}$.

The study of the boundedness of weighted Bergman projections on $L^{p}$-spaces is a compelling topic that has attracted a considerable amount of attention during the last decades. A well known result due to Bekollé and Bonami $[4,5]$ describes the weights $\omega$ such that the Bergman projection $P_{\eta}$, induced by the standard weight $(\eta+1)\left(1-|z|^{2}\right)^{\eta}$, is bounded on $L_{\omega}^{q}$ for $1<q<\infty$. We denote this class of weights by $B_{q}(\eta)$, and write $B_{q}=\cup_{\eta>-1} B_{q}(\eta)$ for short. In the case of a standard weight, the Bergman reproducing kernels are given by the neat formula $(1-\bar{z} \zeta)^{-(2+\eta)}$. However, for a general radial weight $v$ the Bergman reproducing kernels $B_{z}^{v}$ may have zeros [18] and such explicit formulas for the kernels do not necessarily exist. This is one of the main obstacles in dealing with $P_{v}[9,16]$. Nonetheless, we shall prove in Proposition 6 below that if $v \in B_{q}$ is radial, then $P_{v}: L_{v}^{q} \rightarrow L_{v}^{q}$ is bounded for each $1<q<\infty$. The proof of this relies on accurate estimates for the integral means of $B_{v}^{z}$ recently obtained in [16, Theorem 1], and the result itself plays an important role in the proof of the main discovery of this paper.

All the above makes the class of radial weights in $B_{q}$ an appropriate framework for the study of the big Hankel operator

$$
H_{f}^{v}(g)(z)=\left(I-P_{v}\right)(f g)(z), \quad f \in L_{v}^{1}, \quad z \in \mathbb{D},
$$

on weighted Bergman spaces. For an analytic function $f$, the Hankel operator $H \frac{\beta}{f}$, induced by a standard projection, has been widely studied on Bergman spaces since the pioneering work of Axler [3], which was later extended in [1]. In the case of a rapidly decreasing weight $v$ and $f \in \mathcal{H}(\mathbb{D})$, Galanopoulos and Pau [10] did an extensive research on $H_{\frac{v}{f}}$ on $A_{v}^{2}$; see [2] for further results. For general symbols, Zhu [21] was the first to build up a bridge between Hankel operators and the mean oscillation of the symbols in the Bergman metric, and this idea has been further developed in several contexts [6-8,22]; see [23] and the references therein for further information on the theory of Hankel operators. More recently, Pau et al. [12] described the complex valued symbols $f$ such that the Hankel operators $H_{f}^{\beta}$ and $H_{\bar{f}}^{\beta}$ are simultaneously bounded from $A_{\alpha}^{p}$ to $L_{\beta}^{q}$, provided $1<p \leq q<\infty$. Our primary aim is to extend this last-mentioned result to the context of radial $B_{q}$-weights. To do this, some definitions are needed. For a radial weight $\omega$, we assume throughout the paper that $\widehat{\omega}(z)=\int_{|z|}^{1} \omega(s) d s>0$ for all $z \in \mathbb{D}$, for otherwise the Bergman space $A_{\omega}^{p}$ would contain 
all analytic functions in $\mathbb{D}$. A radial weight $\omega$ belongs to the class $\widehat{\mathcal{D}}$ if there exists a constant $C=C(\omega)>1$ such that $\widehat{\omega}(r) \leq C \widehat{\omega}\left(\frac{1+r}{2}\right)$ for all $0 \leq r<1$. Moreover, if there exist $K=K(\omega)>1$ and $C=C(\omega)>1$ such that

$$
\widehat{\omega}(r) \geq C \widehat{\omega}\left(1-\frac{1-r}{K}\right), \quad 0 \leq r<1,
$$

then $\omega \in \check{\mathcal{D}}$. We write $\mathcal{D}=\widehat{\mathcal{D}} \cap \breve{\mathcal{D}}$ for short. For basic properties of these classes of weights and more, see $[13,14]$ and the references therein. Let $\beta(z, \zeta)$ denote the hyperbolic distance between $z, \zeta \in \mathbb{D}, \Delta(z, r)$ the hyperbolic disc of center $z$ and radius $r>0$, and $S(z)$ the Carleson square associated to $z$. For $0<p, q<\infty$ and radial weights $\omega, v$, define

$$
\gamma(z)=\gamma_{\omega, v, p, q}(z)=\frac{\widehat{v}(z)^{\frac{1}{q}}(1-|z|)^{\frac{1}{q}}}{\widehat{\omega}(z)^{\frac{1}{p}}(1-|z|)^{\frac{1}{p}}}, \quad z \in \mathbb{D} .
$$

Further, for $f \in L_{v, \text { loc }}^{1}$, write $\widehat{f}_{r, v}(z)=\frac{\int_{\Delta(z, r)} f(\zeta) v(\zeta) d A(\zeta)}{v(\Delta(z, r))}$ and

$$
\mathrm{MO}_{v, q, r}(f)(z)=\left(\frac{1}{v(\Delta(z, r))} \int_{\Delta(z, r)}\left|f(\zeta)-\widehat{f}_{r, v}(z)\right|^{q} v(\zeta) d A(\zeta)\right)^{\frac{1}{q}}
$$

for all $z \in \mathbb{D}$. It is worth noticing that for prefixed $r>0$, the quantity $v(\Delta(z, r))$ may equal to zero for some $z$ arbitrarily close to the boundary if $v \in \widehat{\mathcal{D}}$. However, if $v \in \mathcal{D}$, then there exists $r_{0}=r_{0}(v)>0$ such that $v(\Delta(z, r)) \asymp v(S(z))>0$ for all $z \in \mathbb{D}$ if $r \geq r_{0}$. The space $\operatorname{BMO}(\Delta)_{\omega, v, p, q, r}$ consists of $f \in L_{\nu, \text { loc }}^{q}$ such that

$$
\|f\|_{\mathrm{BMO}(\Delta)_{\omega, v, p, q, r}}=\sup _{z \in \mathbb{D}}\left(\mathrm{MO}_{v, q, r}(f)(z) \gamma(z)\right)<\infty .
$$

We will show that if $v \in \mathcal{D}$, then $\operatorname{BMO}(\Delta)_{\omega, v, p, q, r}$ does not depend on $r$ for $r \geq r_{0}$. In this case, we simply write $\operatorname{BMO}(\Delta)_{\omega, v, p, q}$. The main result of this study reads as follows and it will be proved in Sect. 5 .

Theorem 1 Let $1<p \leq q<\infty, \omega \in \mathcal{D}, v \in B_{q}$ a radial weight and $f \in L_{v}^{q}$. Then $H_{f}^{v}, H_{f}^{v}: A_{\omega}^{p} \rightarrow L_{v}^{q}$ are bounded if and only if $f \in \operatorname{BMO}(\Delta)_{\omega, v, p, q}$.

The approach employed in the proof of this result follows the guideline of [12, Thorem 4.1], however a good number of steps cannot be adapted straightforwardly and need substantial modifications. In Sect. 2 we prove some results concerning the classes of weights involved in this work and the boundedness of the Bergman projection $P_{v}$, while in Sect. 3 we introduce and study two spaces of functions on $\mathbb{D}$. One of them is denoted as $\operatorname{BA}(\Delta)_{\omega, v, p, q}$, and although its initial definition depends on $r$, it can be described in terms of an appropriate Berezin transform or simply observing that $f \in \mathrm{BA}(\Delta)_{\omega, v, p, q}$ if and only the multiplication operator $M_{f}(g)=f g$ is bounded from $A_{\omega}^{p}$ to $L_{v}^{q}$ [15]. The second one, denoted by $\operatorname{BO}(\Delta)_{\omega, v, p, q}$, consists of continuous functions on $\mathbb{D}$ such that the oscillation in the Bergman metric is bounded in terms of the auxiliary function $\gamma$ given in (1.2). We show that $f \in \mathrm{BO}(\Delta)_{\omega, \nu, p, q}$ if and only if

$$
|f(z)-f(\zeta)| \lesssim\|f\|_{\mathrm{BO}(\Delta)_{\omega, v, p, q}}(1+\beta(z, \zeta)) \Gamma_{\tau}(z, \zeta) \quad z, \zeta \in \mathbb{D}
$$


where

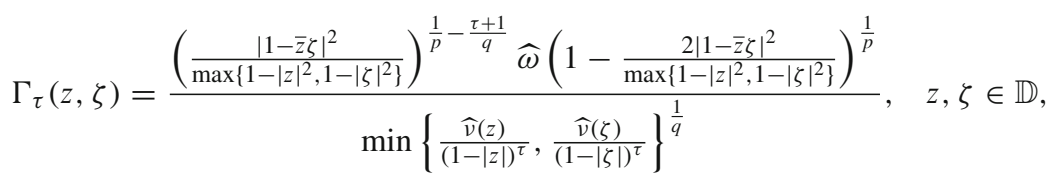

for an appropriate (small) constant $\tau=\tau(\omega, \nu)>0$. If $\omega$ and $v$ are standard weights, then $\Gamma_{\tau}$ does not coincide with the function playing the corresponding role in [12, Lemma 3.2]; in the latter case the function is simpler in many aspects and does not depend on the additional parameter $\tau$. Then, we show that

$$
\operatorname{BMO}(\Delta)_{\omega, v, p, q}=\operatorname{BA}(\Delta)_{\omega, v, p, q}+\operatorname{BO}(\Delta)_{\omega, v, p, q} .
$$

In order to prove this decomposition, due to the complex nature of $\Gamma_{\tau}(z, \zeta)$, we are forced to split $\mathbb{D}$ into several regions depending on $z$, establish sharp estimates for $\Gamma_{\tau}(z, \zeta)$ in each region and then apply properties of weights in $\mathcal{D}$. The identity (1.3) together with a description of the boundedness of the integral operator

$$
T_{b, c} f(z)=\int_{\mathbb{D}} f(\zeta) \frac{\left(1-|\zeta|^{2}\right)^{b}}{(1-z \bar{\zeta})^{c}} d A(\zeta)
$$

and its maximal counterpart from $A_{\omega}^{p}$ to $L_{v}^{q}$, see Sect. 4 below, are key tools to prove that each $f \in \operatorname{BMO}(\Delta)_{\omega, v, p, q}$ induces a bounded Hankel operator from $A_{\omega}^{p}$ to $L_{v}^{q}$. Theorem 1 will be proved in Sect. 5 .

Finally, in Sect. 6, as a byproduct of Theorem 1, we describe the analytic symbols such that $H_{\bar{f}}: A_{\omega}^{p} \rightarrow L_{v}^{q}$ is bounded. The space $\mathcal{B}_{d \gamma}$ consists of $f \in \mathcal{H}(\mathbb{D})$ such that

$$
\|f\|_{\mathcal{B}_{d \gamma}}=\sup _{z \in \mathbb{D}}\left|f^{\prime}(z)\right|(1-|z|) \gamma(z)+|f(0)|<\infty,
$$

where $\gamma$ is given by (1.2).

Theorem 2 Let $1<p \leq q<\infty, \omega \in \mathcal{D}, v \in B_{q}$ a radial weight and $f \in A_{v}^{1}$. Then $H \frac{v}{f}: A_{\omega}^{p} \rightarrow L_{v}^{q}$ is bounded if and only if $f \in \mathcal{B}_{d \gamma}$.

Throughout the paper $\frac{1}{p}+\frac{1}{p^{\prime}}=1$ for $1<p<\infty$. Further, the letter $C=C(\cdot)$ will denote an absolute constant whose value depends on the parameters indicated in the parenthesis, and may change from one occurrence to another. We will use the notation $a \lesssim b$ if there exists a constant $C=C(\cdot)>0$ such that $a \leq C b$, and $a \gtrsim b$ is understood in an analogous manner. In particular, if $a \lesssim b$ and $a \gtrsim b$, then we will write $a \asymp b$.

\section{Auxiliary results}

For a radial weight $\omega, K>1$ and $0 \leq r<1$, let $\rho_{n}^{r}=\rho_{n}^{r}(\omega, K)$ be defined by $\widehat{\omega}\left(\rho_{n}^{r}\right)=$ $\widehat{\omega}(r) K^{-n}$ for all $n \in \mathbb{N} \cup\{0\}$. Write $\rho_{n}=\rho_{n}^{0}$ for short. For $x \geq 1$, write $\omega_{x}=\int_{0}^{1} r^{x} \omega(r) d r$. Denote

$$
\omega^{\star}(z)=\int_{|z|}^{1} \log \frac{s}{|z|} \omega(s) s d s, \quad z \in \mathbb{D} \backslash\{0\} .
$$

Throughout the proofs we will repeatedly use several basic properties of weights in the classes $\widehat{\mathcal{D}}$ and $\breve{\mathcal{D}}$. For a proof of the first lemma, see [13, Lemma 2.1]; the second one can be proved by similar arguments. 
Lemma A Let $\omega$ be a radial weight. Then the following statements are equivalent:

(i) $\omega \in \widehat{\mathcal{D}}$;

(ii) There exist $C=C(\omega)>0$ and $\beta=\beta(\omega)>0$ such that

$$
\widehat{\omega}(r) \leq C\left(\frac{1-r}{1-t}\right)^{\beta} \widehat{\omega}(t), \quad 0 \leq r \leq t<1 ;
$$

(iii) There exist $C=C(\omega)>0$ and $\gamma=\gamma(\omega)>0$ such that

$$
\int_{0}^{t}\left(\frac{1-t}{1-s}\right)^{\gamma} \omega(s) d s \leq C \widehat{\omega}(t), \quad 0 \leq t<1 ;
$$

(iv) There exists $\lambda=\lambda(\omega) \geq 0$ such that

$$
\int_{\mathbb{D}} \frac{d A(z)}{|1-\bar{\zeta} z|^{\lambda+1}} \asymp \frac{\widehat{\omega}(\zeta)}{(1-|\zeta|)^{\lambda}}, \quad \zeta \in \mathbb{D} ;
$$

(v) There exist $K=K(\omega)>1$ and $C=C(\omega, K)>1$ such that $1-\rho_{n}^{r}(\omega, K) \geq$ $C\left(1-\rho_{n+1}^{r}(\omega, K)\right)$ for some (equivalently for all) $0 \leq r<1$ and for all $n \in \mathbb{N} \cup\{0\}$.

Lemma B Let $\omega$ be a radial weight. Then $\omega \in \check{\mathcal{D}}$ if and only if there exist $C=C(\omega)>0$ and $\alpha=\alpha(\omega)>0$ such that

$$
\widehat{\omega}(t) \leq C\left(\frac{1-t}{1-r}\right)^{\alpha} \widehat{\omega}(r), \quad 0 \leq r \leq t<1 .
$$

Two more results on weights of more general nature than Lemmas A and B are also needed.

Lemma 3 Let $\omega$ be a radial weight. Then the following statements are equivalent:

(i) $\omega \in \widehat{\mathcal{D}}$;

(ii) For some (equivalently for each) $v \in \mathcal{D}$ there exists a constant $C=C(\omega, v)>0$ such that

$$
\int_{r}^{1} \frac{\omega(t) \widehat{v}(t)}{\widehat{\omega}(t)} d t \leq C \widehat{v}(r), \quad 0 \leq r<1
$$

(iii) For some (equivalently for each) $v \in \mathcal{D}$ there exists a constant $C=C(\omega, v)>0$ such that

$$
\int_{0}^{r} \frac{\omega(t)}{\widehat{\omega}(t) \widehat{v}(t)} d t \leq \frac{C}{\widehat{v}(r)}, \quad 0 \leq r<1 .
$$

Proof Let first $\omega \in \widehat{\mathcal{D}}$ and $0 \leq r<1$, and consider $\rho_{n}^{r}=\rho_{n}^{r}(\omega, K)$ for all $n \in \mathbb{N} \cup\{0\}$. Then Lemma $\mathrm{B}$, applied to $v \in \mathcal{D} \subset \breve{\mathcal{D}}$, and Lemma $\mathrm{A}(\mathrm{v})$, applied to $\omega$, imply

$$
\begin{aligned}
\int_{r}^{1} \frac{\omega(t) \widehat{v}(t)}{\widehat{\omega}(t)} d t & =\sum_{n=0}^{\infty} \int_{\rho_{n}^{r}}^{\rho_{n+1}^{r}} \frac{\omega(t) \widehat{v}(t)}{\widehat{\omega}(t)} d t \leq \sum_{n=0}^{\infty} \widehat{v}\left(\rho_{n}^{r}\right) \int_{\rho_{n}^{r}}^{\rho_{n+1}^{r}} \frac{\omega(t)}{\widehat{\omega}(t)} d t \\
& \lesssim \log K \frac{\widehat{v}\left(\rho_{0}^{r}\right)}{\left(1-\rho_{0}^{r}\right)^{\beta}} \sum_{n=0}^{\infty}\left(1-\rho_{n}^{r}\right)^{\beta} \\
& \leq \widehat{v}(r) \log K \sum_{n=0}^{\infty} \frac{1}{\left(C^{\beta}\right)^{n}}=\widehat{v}(r) \log K \frac{C^{\beta}}{C^{\beta}-1}, \quad 0 \leq r<1,
\end{aligned}
$$


for a suitably fixed $K=K(\omega)>1$, and thus (ii) is satisfied. Conversely, (ii) implies

$$
C \widehat{v}(r) \geq \int_{r}^{1} \frac{\omega(t) \widehat{v}(t)}{\widehat{\omega}(t)} d t \geq \int_{r}^{\frac{1+r}{2}} \frac{\omega(t) \widehat{v}(t)}{\widehat{\omega}(t)} d t \geq \widehat{v}\left(\frac{1+r}{2}\right) \log \frac{\widehat{\omega}(r)}{\widehat{\omega}\left(\frac{1+r}{2}\right)}, \quad 0 \leq r<1,
$$

and since $v \in \mathcal{D} \subset \widehat{\mathcal{D}}$ by the hypothesis, we deduce $\widehat{\omega}(r) \lesssim \widehat{\omega}\left(\frac{1+r}{2}\right)$ for all $0 \leq r<1$. Thus $\omega \in \widehat{\mathcal{D}}$.

Let $\omega \in \widehat{\mathcal{D}}$ and $0 \leq r<1$, and consider $\rho_{n}=\rho_{n}(\omega, K)$ for all $n \in \mathbb{N} \cup\{0\}$. Fix $k=k(\omega, K) \in \mathbb{N} \cup\{0\}$ such that $\rho_{k} \leq r<\rho_{k+1}$. Then

$$
\int_{0}^{r} \frac{\omega(t)}{\widehat{\omega}(t) \widehat{v}(t)} d t=\sum_{n=0}^{k-1} \int_{\rho_{n}}^{\rho_{n+1}} \frac{\omega(t)}{\widehat{\omega}(t) \widehat{v}(t)} d t+\int_{\rho_{k}}^{r} \frac{\omega(t)}{\widehat{\omega}(t) \widehat{v}(t)} d t, \quad 0 \leq r<1,
$$

where, by Lemma $\mathrm{B}$, applied to $v \in \mathcal{D} \subset \check{\mathcal{D}}$, and Lemma $\mathrm{A}(\mathrm{v})$, applied to $\omega$,

$$
\begin{aligned}
\sum_{n=0}^{k-1} \int_{\rho_{n}}^{\rho_{n+1}} \frac{\omega(t)}{\widehat{\omega}(t) \widehat{v}(t)} d t & \leq \sum_{n=0}^{k-1} \frac{1}{\widehat{v}\left(\rho_{n+1}\right)} \int_{\rho_{n}}^{\rho_{n+1}} \frac{\omega(t)}{\widehat{\omega}(t)} d t \\
& \lesssim \sum_{n=0}^{k-1} \frac{\left(1-\rho_{k}\right)^{\alpha}}{\widehat{v}\left(\rho_{k}\right)} \frac{1}{\left(1-\rho_{n+1}\right)^{\alpha}} \log \left(\frac{\widehat{\omega}\left(\rho_{n}\right)}{\widehat{\omega}\left(\rho_{n+1}\right)}\right) \\
& \leq \log K \frac{\left(1-\rho_{k}\right)^{\alpha}}{\widehat{v}(r)} \sum_{n=0}^{k-1} \frac{1}{\left(C^{\alpha}\right)^{k-1-n}\left(1-\rho_{k}\right)^{\alpha}} \\
& \leq \frac{\log K}{\widehat{v}(r)} \sum_{n=0}^{\infty} \frac{1}{\left(C^{\alpha}\right)^{n}}=\frac{\log K}{\widehat{v}(r)} \frac{C^{\alpha}}{C^{\alpha}-1}, \quad k \in \mathbb{N},
\end{aligned}
$$

for some $\alpha=\alpha(v)>0$ and for a suitably fixed $K=K(\omega)>1$, and similarly,

$$
\int_{\rho_{k}}^{r} \frac{\omega(t)}{\widehat{\omega}(t) \widehat{v}(t)} d t \leq \frac{1}{\widehat{v}(r)} \log \left(\frac{\widehat{\omega}\left(\rho_{k}\right)}{\widehat{\omega}(r)}\right) \leq \frac{\log K}{\widehat{v}(r)}, \quad k \in \mathbb{N} \cup\{0\} .
$$

The statement (iii) follows from these estimates.

Conversely, by replacing $r$ by $\frac{1+r}{2}$ in (iii) we obtain

$$
\frac{C}{\widehat{v}\left(\frac{1+r}{2}\right)} \geq \int_{0}^{(1+r) / 2} \frac{\omega(t)}{\widehat{\omega}(t) \widehat{v}(t)} d t \geq \int_{r}^{(1+r) / 2} \frac{\omega(t)}{\widehat{\omega}(t) \widehat{v}(t)} d t \geq \frac{1}{\widehat{v}(r)} \log \frac{\widehat{\omega}(r)}{\widehat{\omega}\left(\frac{1+r}{2}\right)}, \quad 0 \leq r<1,
$$

and since $v \in \mathcal{D} \subset \widehat{\mathcal{D}}$ by the hypothesis, we deduce $\widehat{\omega}(r) \lesssim \widehat{\omega}\left(\frac{1+r}{2}\right)$ for all $0 \leq r<1$. Thus $\omega \in \widehat{\mathcal{D}}$.

Lemma 4 Let $\omega, v \in \mathcal{D}$, and denote $\sigma=\sigma_{\omega, v}=\omega \widehat{v} / \widehat{\omega}$. Then $\widehat{\sigma} \asymp \widehat{v}$ on $[0,1)$, and hence $\sigma \in \mathcal{D}$

Proof Lemma 3(ii) implies $\widehat{\sigma} \lesssim \widehat{v}$ on $[0,1)$. The argument used to prove (i) $\Rightarrow$ (ii) in the said lemma shows that $\widehat{\sigma} \gtrsim \widehat{v}$ on $[0,1)$, provided $\omega \in \check{\mathcal{D}}$ and $v \in \mathcal{D}$. Thus $\widehat{\sigma} \asymp \widehat{v}$, and $\sigma \in \mathcal{D}$ by Lemmas A(ii) and B.

The next lemma says that in many instances concerning $A^{p}$-norms we may replace $\omega$ by $\widetilde{\omega}=\widehat{\omega} /(1-|\cdot|)$ if $\omega \in \mathcal{D}$. This result has the flavor of radial Carleson measures and indeed can be established by appealing to the characterization of Carleson measures for the Bergman space $A_{\omega}^{p}$ induced by $\omega \in \widehat{\mathcal{D}}$ given in [15]. That approach requires showing that the involved 
weights belong to $\widehat{\mathcal{D}}$, which is of course the case, and thus involves more calculations than the simple proof given below.

Lemma 5 Let $0<p<\infty, \omega \in \mathcal{D}$ and $-\alpha<\kappa<\infty$, where $\alpha=\alpha(\omega)>0$ is that of Lemma B. Then

$$
\int_{\mathbb{D}}|f(z)|^{p}(1-|z|)^{\kappa} \omega(z) d A(z) \asymp \int_{\mathbb{D}}|f(z)|^{p}(1-|z|)^{\kappa-1} \widehat{\omega}(z) d A(z), \quad f \in \mathcal{H}(\mathbb{D}) .
$$

Proof The function $(1-|\cdot|)^{\kappa-1} \widehat{\omega}$ is a weight for each $\kappa>-\alpha$ by Lemma B. Therefore an integration by parts shows that (2.1) is equivalent to

$\int_{0}^{1} \frac{\partial}{\partial r} M_{p}^{p}(r, f)\left(\int_{r}^{1}(1-t)^{\kappa} \omega(t) d t\right) d r \asymp \int_{0}^{1} \frac{\partial}{\partial r} M_{p}^{p}(r, f)\left(\int_{r}^{1}(1-t)^{\kappa-1} \widehat{\omega}(t) d t\right) d r$.

Another integration by parts reveals that both integrals from $r$ to 1 above are bounded by a constant times $\widehat{\omega}(r)(1-r)^{\kappa}$. But Lemma A(ii) implies

$\int_{r}^{1}(1-t)^{\kappa-1} \widehat{\omega}(t) d t \gtrsim \frac{\widehat{\omega}(r)}{(1-r)^{\beta(\omega)}} \int_{r}^{1}(1-t)^{\kappa-1+\beta(\omega)} d t \asymp \widehat{\omega}(r)(1-r)^{\kappa}, \quad 0 \leq r<1$,

and

$\int_{r}^{1}(1-t)^{\kappa} \omega(t) d t \gtrsim \frac{\widehat{\omega}(r)}{(1-r)^{\beta(\omega)}} \int_{r}^{1} \frac{\omega(t)(1-t)^{\kappa+\beta(\omega)}}{\widehat{\omega}(t)} d t \asymp \widehat{\omega}(r)(1-r)^{\kappa}, \quad 0 \leq r<1$,

by Lemma 4 . The assertion follows.

The last auxiliary results shows that each radial weight in the Bekollé-Bonami class $B_{q}$ belongs to $\mathcal{D}$, and for each $v \in \mathcal{D}$ the maximal Bergman projection

$$
P_{v}^{+}(f)(z)=\int_{\mathbb{D}} f(\zeta)\left|B_{z}^{v}(\zeta)\right| v(\zeta) d A(\zeta), \quad z \in \mathbb{D},
$$

is bounded on $L_{v}^{q}$. It is worth noticing that obviously $\mathcal{D} \not \subset \cup_{1<q<\infty} B_{q}$ because $v \in \mathcal{D}$ may vanish on a set of positive measure.

Proposition 6 Let $1<q<\infty$ and $v \in B_{q}$ a radial weight. Then $v \in \mathcal{D}$. Moreover, $P_{v}^{+}: L_{v}^{q} \rightarrow L_{v}^{q}$ is bounded for all $v \in \mathcal{D}$.

Proof If $v \in B_{q}$, then by [5] there exists $\beta>-1$ such that

$$
\left(\int_{S(a)} v(z) d A(z)\right)^{\frac{1}{q}}\left(\int_{S(a)}\left(\frac{(1-|z|)^{\beta}}{v(z)}\right)^{\frac{q^{\prime}}{q}}(1-|z|)^{\beta} d A(z)\right)^{\frac{1}{q^{\prime}}} \lesssim(1-|a|)^{(2+\beta)}, \quad a \in \mathbb{D} .
$$

Since $v$ is radial, this condition easily implies $v \in \mathcal{D}$.

Let now $1<q<\infty$ and $v \in \mathcal{D}$, and define $h=\widehat{v}^{-\frac{1}{q q^{\prime}}}$. Then $\int_{t}^{1} h(s)^{q^{\prime}} v(s) d s \asymp \widehat{v}(t)^{\frac{1}{q^{\prime}}}$ for all $0 \leq t<1$. Therefore Lemma B yields

$$
\int_{0}^{r} \frac{\int_{t}^{1} h(s)^{q^{\prime}} v(s) d s}{\widehat{v}(t)(1-t)} d t \asymp \int_{0}^{r} \frac{d t}{\widehat{v}(t)^{\frac{1}{q}}(1-t)} \lesssim \frac{1}{\widehat{v}(r)^{\frac{1}{q}}}=h^{q^{\prime}}(r), \quad 0 \leq r<1 .
$$


Moreover, by symmetry, (2.2) with $q^{\prime}$ in place of $q$ is satisfied. Since $v \in \widehat{\mathcal{D}}$, we may apply [16, Theorem 1] and (2.2) to deduce

$$
\int_{\mathbb{D}}\left|B_{z}^{v}(\zeta)\right| h^{p^{\prime}}(\zeta) v(\zeta) d A(\zeta) \lesssim h^{p^{\prime}}(z), \quad z \in \mathbb{D},
$$

and

$$
\int_{\mathbb{D}}\left|B_{z}^{v}(\zeta)\right| h^{p}(z) v(z) d A(z) \lesssim h^{p}(\zeta), \quad \zeta \in \mathbb{D} .
$$

It follows from Schur's test [23, Theorem 3.6] that the maximal Bergman projection $P_{v}^{+}$: $L_{v}^{p} \rightarrow L_{v}^{p}$ is bounded.

\section{Some spaces of functions}

Recall that

$$
\gamma(z)=\gamma_{\omega, v, p, q}(z)=\frac{\widehat{v}(z)^{\frac{1}{q}}(1-|z|)^{\frac{1}{q}}}{\widehat{\omega}(z)^{\frac{1}{p}}(1-|z|)^{\frac{1}{p}}}, \quad z \in \mathbb{D},
$$

and $\widehat{f}_{r, v}(z)=\frac{\int_{\Delta(z, r)} f(\zeta) v(\zeta) d A(\zeta)}{v(\Delta(z, r))}$ for $f \in L_{\nu, \text { loc }}^{1}$, and

$$
\mathrm{MO}_{v, q, r}(f)(z)=\left(\frac{1}{v(\Delta(z, r))} \int_{\Delta(z, r)}\left|f(\zeta)-\widehat{f}_{r, v}(z)\right|^{q} \nu(\zeta) d A(\zeta)\right)^{\frac{1}{q}}
$$

for all $z \in \mathbb{D}$. If $v \in \check{\mathcal{D}}$, then by the definition there exist $K=K(v)>1$ and $C=C(v)>1$ such that

$$
\int_{r}^{1-\frac{1-r}{K}} v(s) d s \geq(C-1) \widehat{v}\left(1-\frac{1-r}{K}\right)>0, \quad 0 \leq r<1 .
$$

It follows that there exists $r_{v} \in(0, \infty)$ such that $v(\Delta(z, r))>0$ for all $z \in \mathbb{D}$ if $r \geq r_{v}$.

The space $\operatorname{BMO}(\Delta)=\operatorname{BMO}(\Delta)_{\omega, v, p, q, r}$ consists of $f \in L_{v, \text { loc }}^{q}$ such that

$$
\|f\|_{\mathrm{BMO}(\Delta)}=\sup _{z \in \mathbb{D}}\left(\mathrm{MO}_{\nu, q, r}(f)(z) \gamma(z)\right)<\infty .
$$

The following lemma is easy to establish; see [12, Lemma 3.1] for a similar result.

Lemma 7 Let $1 \leq p, q<\infty, \omega$ a radial weight, $v \in \check{\mathcal{D}}$ and $r_{v} \leq r<\infty$. Then

$\mathrm{MO}_{v, q, r}(f)(z) \leq 2\left(\frac{1}{v(\Delta(z, r))} \int_{\Delta(z, r)}|f(\zeta)-\lambda|^{q} v(\zeta) d A(\zeta)\right)^{\frac{1}{q}}, \quad z \in \mathbb{D}, \lambda \in \mathbb{C}, f \in L_{v}^{q}$,

and therefore $f \in L_{v}^{q}$ belongs to $\operatorname{BMO}(\Delta)$ if and only if for each $z \in \mathbb{D}$ there exists $\lambda_{z} \in \mathbb{C}$ such that

$$
\sup _{z \in \mathbb{D}}\left(\frac{\gamma(z)^{q}}{\nu(\Delta(z, r))} \int_{\Delta(z, r)}\left|f(\zeta)-\lambda_{z}\right|^{q} \nu(\zeta) d A(\zeta)\right)<\infty
$$


For $0<p, q<\infty, 0 \leq \tau<\infty$ and radial weights $\omega, v$, let

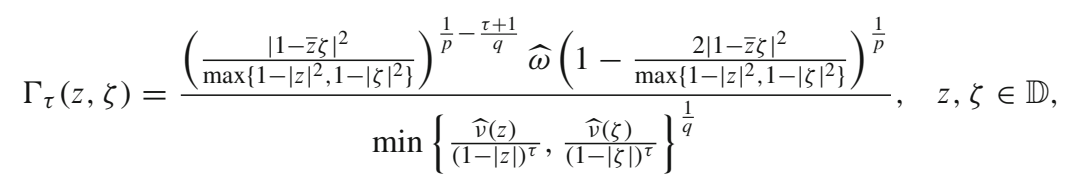

with the understanding that $\widehat{\omega}(t)=\widehat{\omega}(0)$ when $t<0$. The following lemma explains the behavior of $\Gamma_{\tau}$ near the diagonal.

Lemma 8 Let $0<p, q, r<\infty, 0 \leq \tau<\infty$ and $\omega, v \in \widehat{\mathcal{D}}$. Then

$$
\Gamma_{\tau}(z, \zeta) \asymp \gamma(z)^{-1} \asymp \gamma(\zeta)^{-1}, \quad \beta(z, \zeta) \leq r .
$$

Proof Clearly

$$
|1-\bar{z} \zeta| \asymp 1-|z| \asymp 1-|\zeta|, \quad \beta(z, \zeta) \leq r,
$$

and hence there exist $0<m_{r}<1<M_{r}<\infty$ such that

$$
m_{r}(1-|z|) \leq \frac{2|1-\bar{z} \zeta|^{2}}{\max \left\{1-|z|^{2}, 1-|\zeta|^{2}\right\}} \leq M_{r}(1-|z|), \quad \beta(z, \zeta) \leq r .
$$

Since $\omega \in \widehat{\mathcal{D}}$ by the hypothesis, and $\widehat{\omega}(t)=\widehat{\omega}(0)$ for $t<0$, Lemma A(ii) implies

$$
\widehat{\omega}(z) \leq \frac{C}{m_{r}^{\beta}} \widehat{\omega}\left(1-m_{r}(1-|z|)\right) \leq \frac{C}{m_{r}^{\beta}} \widehat{\omega}\left(1-\frac{2|1-\bar{z} \zeta|^{2}}{\max \left\{1-|z|^{2}, 1-|\zeta|^{2}\right\}}\right), \quad \beta(z, \zeta) \leq r,
$$

and

$\widehat{\omega}\left(1-\frac{2|1-\bar{z} \zeta|^{2}}{\max \left\{1-|z|^{2}, 1-|\zeta|^{2}\right\}}\right) \leq C M_{r}^{\beta} \widehat{\omega}\left(1-M_{r}(1-|z|)\right) \leq C M_{r}^{\beta} \widehat{\omega}(z), \quad \beta(z, \zeta) \leq r$,

for some $C=C(\omega)>0$ and $\beta=\beta(\omega)>0$. Further, $\widehat{v}(z) \asymp \widehat{v}(\zeta)$ and $\widehat{\omega}(z) \asymp \widehat{\omega}(\zeta)$ if $\beta(z, \zeta) \leq r$ by Lemma $\mathrm{A}(\mathrm{ii})$. The assertion follows from these estimates.

For continuous $f: \mathbb{D} \rightarrow \mathbb{C}$ and $0<r<\infty$, define

$$
\Omega_{r} f(z)=\sup \{|f(z)-f(\zeta)|: \beta(z, \zeta)<r\}, \quad z \in \mathbb{D},
$$

and let $\mathrm{BO}(\Delta)=\mathrm{BO}(\Delta)_{\omega, \nu, p, q, r}$ denote the space of those $f$ such that

$$
\|f\|_{\mathrm{BO}(\Delta)}=\sup _{z \in \mathbb{D}}\left(\Omega_{r} f(z) \gamma(z)\right)<\infty .
$$

Lemma 9 shows that the space $\mathrm{BO}(\Delta)=\mathrm{BO}(\Delta)_{\omega, \nu, p, q, r}$ is independent of $r$.

Lemma 9 Let $0<p \leq q<\infty, 0<r<\infty, \omega, v \in \check{\mathcal{D}}$ and $\gamma(z)=\gamma_{\omega, \nu, p, q}(z)=$ $\frac{\widehat{v}(z)^{\frac{1}{q}}(1-|z|)^{\frac{1}{q}}}{\widehat{\omega}(z)^{\frac{1}{p}}(1-|z|)^{\frac{1}{p}}}$. Let $f: \mathbb{D} \rightarrow \mathbb{C}$ be continuous, and $0<\tau<\min \{q \alpha(\omega) / p, \alpha(v)\}$, where $\alpha(v)$ and $\alpha(\omega)$ are those from Lemma B. Then the following statements are equivalent:

(i) $f \in \mathrm{BO}(\Delta)$;

(ii) $|f(z)-f(\zeta)| \lesssim\|f\|_{\mathrm{BO}(\Delta)}(1+\beta(z, \zeta)) \Gamma_{\tau}(z, \zeta)$ for all $z, \zeta \in \mathbb{D}$. 
Proof Lemma 8 shows that (ii) implies (i). For the converse, assume (i), that is,

$$
|f(z)-f(\zeta)| \gamma(z) \leq\|f\|_{\mathrm{BO}(\Delta)}, \quad \beta(z, \zeta)<r .
$$

The estimate (ii) for $\beta(z, \zeta) \leq r$ then follows from Lemma 8. If $\beta(z, \zeta)>r$, let $N=$ $\max \{n \in \mathbb{N}: n \leq \beta(z, \zeta) / r+1\}$, and pick up $N+1$ points from the geodesic joining $z$ and $\zeta$ such that $\beta\left(z_{j}, z_{j+1}\right)=\beta(z, \zeta) / N<r$ for all $j=0, \ldots, N-1$. Then, as the hyperbolic distance is additive along geodesics, (3.3) yields

$$
|f(z)-f(\zeta)| \leq \sum_{j=0}^{N-1}\left|f\left(z_{j}\right)-f\left(z_{j+1}\right)\right| \leq\|f\|_{\mathrm{BO}(\Delta)} \sum_{j=0}^{N-1} \frac{\widehat{\omega}\left(z_{j}\right)}{\widehat{v}\left(z_{j}\right)}\left(1-\left|z_{j}\right|\right)^{\frac{1}{p}-\frac{1}{q}} .
$$

Next, observe that

$$
1-\left|z_{j}\right| \leq \frac{2|1-\bar{z} \zeta|^{2}}{\max \left\{1-|z|^{2}, 1-|\zeta|^{2}\right\}}, \quad j=0, \ldots, N
$$

see the proof of [12, Lemma 3.2] for details. This together with the inequality $\frac{1}{p}-\frac{1}{q} \geq 0$ gives

$$
\begin{aligned}
|f(z)-f(\zeta)| & \leq\|f\|_{\mathrm{BO}(\Delta)}\left(\frac{2|1-\bar{z} \zeta|^{2}}{\max \left\{1-|z|^{2}, 1-|\zeta|^{2}\right\}}\right)^{\frac{1}{p}-\frac{1}{q}} \sum_{j=0}^{N-1} \frac{\widehat{\omega}\left(z_{j}\right)^{\frac{1}{p}}}{\widehat{v}\left(z_{j}\right)^{\frac{1}{q}}} \\
& =\|f\|_{\mathrm{BO}(\Delta)}\left(\frac{2|1-\bar{z} \zeta|^{2}}{\max \left\{1-|z|^{2}, 1-|\zeta|^{2}\right\}}\right)^{\frac{1}{p}-\frac{1}{q}} \sum_{j=0}^{N-1} \frac{\widehat{\omega}\left(z_{j}\right)^{\frac{1}{p}}}{\left(1-\left|z_{j}\right|\right)^{\frac{\tau}{q}}} \frac{\left(1-\left|z_{j}\right|\right)^{\frac{\tau}{q}}}{\widehat{v}\left(z_{j}\right)^{\frac{1}{q}}} .
\end{aligned}
$$

The election of $\tau$ together with Lemma B shows that the functions $\widehat{\omega}(r) /(1-r)^{\frac{p \tau}{q}}$ and $\widehat{v}(r) /(1-r)^{\tau}$ are essentially decreasing on $[0,1)$. Therefore the inequalities (3.4) and $\left|z_{j}\right| \leq$ $\max \{|z|,|\zeta|\}$ yield

$$
\begin{aligned}
|f(z)-f(\zeta)| \lesssim & \|f\|_{\mathrm{BO}(\Delta)}\left(\frac{2|1-\bar{z} \zeta|^{2}}{\max \left\{1-|z|^{2}, 1-|\zeta|^{2}\right\}}\right)^{\frac{1}{p}-\frac{\tau+1}{q}} \\
& \cdot \widehat{\omega}\left(1-\frac{2|1-\bar{z} \zeta|^{2}}{\max \left\{1-|z|^{2}, 1-|\zeta|^{2}\right\}}\right)^{\frac{1}{p}} \sum_{j=0}^{N-1} \frac{\left(1-\left|z_{j}\right|\right)^{\frac{\tau}{q}}}{\widehat{v}\left(z_{j}\right)^{\frac{1}{q}}} \\
& \lesssim\|f\|_{\mathrm{BO}(\Delta)} \Gamma_{\tau}(z, \zeta) N \lesssim\|f\|_{\mathrm{BO}(\Delta)}(1+\beta(z, \zeta)) \Gamma_{\tau}(z, \zeta), \quad \beta(z, \zeta)>r .
\end{aligned}
$$

Therefore (ii) is satisfied.

For $0<p, q<\infty, 0<r<\infty$ and radial weights $\omega, v$, the space $\operatorname{BA}(\Delta)=$ $\operatorname{BA}(\Delta)_{\omega, v, p, q, r}$ consists of $f \in L_{v, \text { loc }}^{q}$ such that

$$
\|f\|_{\mathrm{BA}(\Delta)}=\sup _{z \in \mathbb{D}}\left(\left(\frac{1}{v(\Delta(z, r))} \int_{\Delta(z, r)}|f(\zeta)|^{q} v(\zeta) d A(\zeta)\right)^{\frac{1}{q}} \gamma(z)\right)<\infty .
$$

For $c, \sigma \in \mathbb{R}$ and a radial weight $\nu$, the general Berezin transform of $\varphi \in L_{v(1-|\cdot|)^{\sigma}}^{1}$ is defined by

$$
B(\varphi)(z)=B_{v, c, \sigma}(\varphi)(z)=\frac{\left(1-|z|^{2}\right)^{c+1}}{\widehat{v}(z)} \int_{\mathbb{D}} \varphi(\zeta) \frac{\left(1-|\zeta|^{2}\right)^{\sigma}}{|1-z \bar{\zeta}|^{2+c+\sigma}} v(\zeta) d A(\zeta), \quad z \in \mathbb{D} .
$$


The next lemma shows, in particular, that the space $\operatorname{BA}(\Delta)=\operatorname{BA}(\Delta)_{\omega, v, p, q, r}$ is independent of $r$ as long as $r$ is sufficiently large depending on $v \in \mathcal{D}$.

Lemma 10 Let $0<p \leq q<\infty, 0<r<\infty$ and $\omega, v \in \mathcal{D}, \gamma(z)=\gamma_{\omega, v, p, q}(z)=$ $\frac{\widehat{v}(z)^{\frac{1}{q}}(1-|z|)^{\frac{1}{q}}}{\widehat{\omega}(z)^{\frac{1}{p}}(1-|z|)^{\frac{1}{p}}}$. If $f \in L_{\nu}^{q}$, then the following statements are equivalent:

(i) There exists $r_{0}=r_{0}(v)>0$ such that $f \in \mathrm{BA}(\Delta)=\mathrm{BA}(\Delta)_{\omega, v, p, q, r}$ for all $r \geq r_{0}$;

(ii) $|f|^{q} v d A$ is a $q$-Carleson measure for $A_{\omega}^{p}$;

(iii) The identity operator Id $: A_{\omega}^{p} \rightarrow L_{|f|^{q} v}^{q}$ is bounded;

(iv) The multiplication operator $M_{f}(g)=f g$ is bounded from $A_{\omega}^{p}$ to $L_{v}^{q}$;

(v) $\sup _{z \in \mathbb{D}} \gamma(z)^{q} B\left(|f|^{q}\right)(z)<\infty$ for all $\sigma>1-\frac{q}{p}(1+\alpha)$ and $c>\max \left\{-1-\sigma, \frac{q}{p}(1+\right.$ $\beta)-2\}$, where $\alpha=\alpha(\omega)>0$ and $\beta=\beta(\omega)>0$ are those of Lemmas $\mathrm{A}($ ii $)$ and $\mathrm{B}$.

Proof It is obvious that (ii), (iii) and (iv) are equivalent by the definitions. Assume (ii) is satisfied, that is,

$$
\left(\int_{\mathbb{D}}|g(\zeta)|^{q}|f(\zeta)|^{q} \nu(\zeta) d A(\zeta)\right)^{\frac{1}{q}} \lesssim\|g\|_{A_{\omega}^{p}, \quad g \in A_{\omega}^{p}}
$$

For $z \in \mathbb{D}$, let $g_{z}(\zeta)=\left(\frac{1-|z|}{1-\bar{z} \zeta}\right)^{\frac{\lambda+1}{p}}$, where $\lambda=\lambda(\omega)>0$ is that of Lemma A(iv). Further, since $v \in \check{\mathcal{D}}$ by the hypothesis, there exists $r_{v} \in(0, \infty)$ such that $v(\Delta(z, r))>0$ for all $r \geq r_{\nu}$. For $g=g_{z}$ and $r \geq r_{\nu},(3.5)$ yields

$$
\left(\frac{1}{v(\Delta(z, r))} \int_{\Delta(z, r)}|f(\zeta)|^{q} v(\zeta) d A(\zeta)\right)^{\frac{1}{q}} \lesssim \frac{\left\|g_{z}\right\|_{A_{\omega}^{p}}}{v(\Delta(z, r))^{\frac{1}{q}}} \lesssim \frac{(\widehat{\omega}(z)(1-|z|))^{\frac{1}{p}}}{v(\Delta(z, r))^{\frac{1}{q}}}, \quad z \in \mathbb{D}
$$

But since $v \in \mathcal{D}$, applications of Lemmas A(ii) and B show that

$$
v(\Delta(z, r)) \asymp \widehat{v}(z)(1-|z|), \quad z \in \mathbb{D},
$$

if $r$ is sufficiently large. It follows that $f \in \operatorname{BA}(\Delta)=\operatorname{BA}(\Delta)_{\omega, v, p, q, r}$ for all such $r$, and thus (i) is satisfied.

Conversely, if (i) is satisfied, then by using (3.6) we deduce

$$
\left(\int_{\Delta(z, r)}|f(\zeta)|^{q} v(\zeta) d A(\zeta)\right)^{\frac{1}{q}} \lesssim \widehat{\omega}(z)^{\frac{1}{p}}(1-|z|)^{\frac{1}{p}}, \quad z \in \mathbb{D}
$$

Therefore $|f|^{q} v d A$ is a $q$-Carleson measure for $A_{\omega}^{p}$ by [17, Theorem 3].

By integrating only over $\Delta(z, r)$ in (v) and using (3.6) we obtain (i) from (v). To complete the proof of the lemma, it remains to show the converse implication. To do this, pick up a sequence $\left\{a_{j}\right\}$ and $0<r<\infty$ in accordance with [23, Lemma 4.7], and observe that $\widehat{\omega}$ is essentially constant in each hyperbolically bounded region by Lemma A(ii). Then by using (3.6), the hypothesis (i), the election of $c$ and $\sigma$, and finally Lemmas A(ii) and B, we deduce 


$$
\begin{aligned}
\frac{\widehat{v}(z) B\left(|f|^{q}\right)(z)}{\left(1-|z|^{2}\right)^{c+1}} & \lesssim \sum_{j=1}^{\infty} \int_{\Delta\left(a_{j}, r\right)}|f(\zeta)|^{q} \frac{\left(1-|\zeta|^{2}\right)^{\sigma}}{|1-z \bar{\zeta}|^{2+c+\sigma}} v(\zeta) d A(\zeta) \\
& \lesssim \sum_{j=1}^{\infty} \frac{\left(1-\left|a_{j}\right|^{2}\right)^{\sigma}}{\left|1-z \overline{a_{j}}\right|^{2+c+\sigma}} \int_{\Delta\left(a_{j}, r\right)}|f(\zeta)|^{q} v(\zeta) d A(\zeta) \\
& \lesssim \sum_{j=1}^{\infty} \frac{\left(1-\left|a_{j}\right|^{2}\right)^{\sigma+1} \widehat{v}\left(a_{j}\right)}{\left|1-z \overline{a_{j}}\right|^{2+c+\sigma} v\left(\Delta\left(a_{j}, r\right)\right)} \int_{\Delta\left(a_{j}, r\right)}|f(\zeta)|^{q} v(\zeta) d A(\zeta) \\
& \lesssim \sum_{i=1}^{\infty} \frac{\left(1-\left|a_{i}\right|^{2}\right)^{\sigma+1} \widehat{v}\left(a_{i}\right)}{\left|1-z \overline{a_{i}}\right|^{2+c+\sigma} \gamma\left(a_{i}\right)^{q}} \asymp \sum_{j=1}^{\infty} \frac{\left(1-\left|a_{j}\right|^{2}\right)^{\sigma+\frac{q}{p}} \widehat{\omega}\left(a_{j}\right)^{\frac{q}{p}}}{\left|1-z \overline{a_{j}}\right|^{2+c+\sigma}} \\
& \lesssim \int_{\mathbb{D}} \frac{\left(1-|u|^{2}\right)^{\sigma+\frac{q}{p}-2} \widehat{\omega}(u)^{\frac{q}{p}}}{|1-z \bar{u}|^{2+c+\sigma}} d A(u) \\
& \lesssim \int_{0}^{|z|} \frac{\widehat{\omega}(t)^{\frac{q}{p}}}{(1-t)^{c+3-\frac{q}{p}}} d t+\frac{1}{(1-|z|)^{c+\sigma+1}} \int_{|z|}^{1}(1-t)^{\sigma+\frac{q}{p}-2} \widehat{\omega}(t)^{\frac{q}{p}} d t \\
& \lesssim \frac{\widehat{\omega}(|z|)^{\frac{q}{p}}}{(1-|z|)^{c+2-\frac{q}{p}} \asymp \frac{\widehat{v}(z)}{\left(1-|z|^{2}\right)^{c+1} \gamma(z)^{q}}, \quad z \in \mathbb{D},}
\end{aligned}
$$

and thus (v) is satisfied.

With these preparations we are ready to show that $\mathrm{BMO}(\Delta)=\mathrm{BA}(\Delta)+\mathrm{BO}(\Delta)$. This follows from the case (ii) of the next theorem.

Theorem 11 Let $1 \leq p \leq q<\infty, \omega, \nu \in \mathcal{D}, \gamma(z)=\gamma_{\omega, \nu, p, q}(z)=\frac{\widehat{v}(z)^{\frac{1}{q}}(1-|z|)^{\frac{1}{q}}}{\widehat{\omega}(z)^{\frac{1}{p}}(1-|z|)^{\frac{1}{p}}}$ and $f \in L_{\nu}^{q}$. Further, let $r \geq r_{v}, \sigma>0$ and

$$
c>2 \frac{q}{p}(\beta(\omega)+1)+\sigma+\max \{2 \beta(\nu), \gamma(v)\},
$$

where $\beta(\omega), \beta(v), \gamma(v)>0$ are associated to $v$ and $\omega$ via Lemma $\mathrm{A}($ ii), (iii). Then the following statements are equivalent:

(i) There exists $r_{0}=r_{0}(v) \geq r_{v}$ such that $f \in \operatorname{BMO}(\Delta)=\operatorname{BMO}(\Delta)_{\omega, v, p, q, r}$ for all $r \geq r_{0}$

(ii) $f=f_{1}+f_{2}$, where $f_{1} \in \mathrm{BA}(\Delta)$ and $f_{2}=\widehat{f}_{r, v} \in \mathrm{BO}(\Delta)$;

(iii) $\sup _{z \in \mathbb{D}}\left(B\left(\left|f-\widehat{f}_{r, v}(z)\right|^{q}\right) \gamma(z)^{q}\right)<\infty$;

(iv) For each $z \in \mathbb{D}$ there exists $\lambda_{z} \in \mathbb{C}$ such that $\sup _{z \in \mathbb{D}}\left(B\left(\left|f-\lambda_{z}\right|^{q}\right) \gamma(z)^{q}\right)<\infty$.

Proof Obviously, (iii) implies (iv). Next assume (iv). The relation (3.6) shows that there exists $r_{0}=r_{0}(v)>0$ such that

$$
\begin{aligned}
& \frac{1}{v(\Delta(z, r))} \int_{\Delta(z, r)}\left|f(\zeta)-\lambda_{z}\right|^{q} v(\zeta) d A(\zeta) \\
& \quad \lesssim \frac{(1-|z|)^{c+1}}{\widehat{v}(z)} \int_{\mathbb{D}}\left|f(\zeta)-\lambda_{z}\right|^{q} \frac{\left(1-|\zeta|^{2}\right)^{\sigma}}{|1-z \bar{\zeta}|^{2+c+\sigma}} v(\zeta) d A(\zeta), \quad z \in \mathbb{D}, \quad r_{0} \leq r<\infty,
\end{aligned}
$$

which together with Lemma 7 shows that (i) is satisfied.

Assume now (i), and let $f_{2}=\widehat{f}_{r, v}$. Since $f \in L_{\nu}^{q}, q \geq 1$ and $r \geq r_{v}$, the function $f_{2}$ is well defined and continuous. Since $\omega, v \in \mathcal{D}$ by the hypothesis, one may use Lemmas A(ii) 
and $\mathrm{B}$ together with the argument in [12,1651-1652] with minor modifications to show that $f_{2}=\widehat{f}_{r, v} \in \mathrm{BO}(\Delta)$ and $f_{1}=f-\widehat{f}_{r, v} \in \mathrm{BA}(\Delta)$. Thus (ii) is satisfied.

To complete the proof it suffices to show that (ii) implies (iii), so assume $f=f_{1}+f_{2}$, where $f_{1} \in \mathrm{BA}(\Delta)$ and $f_{2}=\widehat{f}_{r, v} \in \mathrm{BO}(\Delta)$. Since $\widehat{f}_{r, v}=\widehat{f}_{1_{r, v}}+\widehat{f}_{2_{r, v}}$, it suffices to prove the condition in (iii) for $f_{1}$ and $f_{2}$ separately. First observe that by Lemma A(iii) the constant function 1 satisfies

$$
B(1)(z) \lesssim \frac{(1-|z|)^{c+1}}{\widehat{v}(z)}\left(\int_{0}^{|z|} \frac{v(t)}{(1-t)^{1+c}} d t+\frac{1}{(1-|z|)^{1+c+\sigma}} \int_{|z|}^{1}(1-t)^{\sigma} v(t) d t\right) \lesssim 1, \quad z \in \mathbb{D},
$$

because $c>\max \{\gamma(v), \sigma\}-1$ by the hypothesis. This together with Hölder's inequality and Lemma 10 yields

$$
\begin{aligned}
B\left(\left|f_{1}-\widehat{f}_{1_{r, v}}(z)\right|^{q}\right) \gamma(z)^{q} & \lesssim\left(B\left(\left|f_{1}\right|^{q}\right)(z)+\left|\widehat{f}_{1_{r, v}}(z)\right|^{q}\right) \gamma(z)^{q} \\
& \leq\left(B\left(\left|f_{1}\right|^{q}\right)(z)+{\widehat{\left|f_{1}\right|_{r, v}^{q}}}_{r}(z)\right) \gamma(z)^{q} \lesssim 1, \quad z \in \mathbb{D},
\end{aligned}
$$

and thus (iii) for $f_{1} \in \mathrm{BA}(\Delta)$ is satisfied.

To deal with $f_{2} \in \mathrm{BO}(\Delta)$, pick up $\tau$ satisfying the hypothesis of Lemma 9. Then

$$
\begin{aligned}
\left|f_{2}(\zeta)-\widehat{f}_{2 r, v}(z)\right| & =\left|\frac{1}{v(\Delta(z, r)} \int_{\Delta(z, r)}\left(f_{2}(\zeta)-f_{2}(u)\right) v(u) d A(u)\right| \\
& \leq \frac{1}{v(\Delta(z, r)} \int_{\Delta(z, r)}\left|f_{2}(\zeta)-f_{2}(u)\right| v(u) d A(u) \\
& \lesssim \frac{1}{v(\Delta(z, r)} \int_{\Delta(z, r)}(1+\beta(\zeta, u)) \Gamma_{\tau}(\zeta, u) v(u) d A(u) \\
& \lesssim(1+\beta(z, \zeta)) \Gamma_{\tau}(z, \zeta), \quad z, \zeta \in \mathbb{D}
\end{aligned}
$$

because $\Gamma_{\tau}(\zeta, u) \asymp \Gamma_{\tau}(z, \zeta)$ for all $u \in \Delta(z, r)$ by Lemma A(ii); see the proof of Lemma 8 for similar estimates. Hence it suffices to show that

$$
\frac{(1-|z|)^{c+1} \gamma(z)^{q}}{\widehat{v}(z)} \int_{\mathbb{D}}\left|(1+\beta(z, \zeta)) \Gamma_{\tau}(z, \zeta)\right|^{q} \frac{\left(1-|\zeta|^{2}\right)^{\sigma}}{|1-z \bar{\zeta}|^{2+c+\sigma}} v(\zeta) d A(\zeta) \lesssim 1, \quad z \in \mathbb{D}
$$

to obtain (iii) for $f_{2} \in \mathrm{BO}(\Delta)$. The proof of (3.7) is involved and will be divided into four separate cases. Before dealing with each case, we observe that since $\beta(z, \zeta)$ grows logarithmically, we may pick up $0<\delta<\min \left\{\sigma, \frac{q}{p} \beta(\omega)+\beta(v)+\frac{\sigma}{2}\right\}$ and a constant $C=C(\delta)>0$ such that

$$
1+\beta(z, \zeta) \leq C \mid\left(1-\left|\varphi_{z}(\zeta)\right|\right)^{-\frac{\delta}{q}}=C\left(\frac{|1-\bar{z} \zeta|^{2}}{(1-|z|)(1-|\zeta|)}\right)^{\frac{\delta}{q}}, \quad z, \zeta \in \mathbb{D}
$$

Case 1 If

$$
\zeta \in D_{1}(z)=\left\{w \in \mathbb{D}: 1-\frac{2|1-z \bar{w}|^{2}}{1-|z|^{2}} \leq 0\right\}
$$


then $1-|z| \lesssim|1-z \bar{\zeta}|^{2}$ and

$$
\begin{aligned}
\Gamma_{\tau}(z, \zeta)^{q} \leq & \frac{\left(\frac{|1-\bar{z} \zeta|^{2}}{\max \left\{1-|z|^{2}, 1-|\zeta|^{2}\right\}}\right)^{\frac{q}{p}-\tau-1}}{\min \left\{\frac{\widehat{v}(z)}{(1-|z|)^{\tau}}, \frac{\widehat{v}(\zeta)}{(1-|\zeta|)^{\tau}}\right\}} \widehat{\omega}(0)^{\frac{q}{p}} \lesssim\left(\frac{|1-z \bar{\zeta}|^{2}}{1-|z|^{2}}\right)^{\frac{q}{p}-\tau-1} \frac{(1-|z|)^{\tau}}{\widehat{v}(z)} \chi_{D(0,|z|)}(\zeta) \\
& +\left(\frac{|1-z \bar{\zeta}|^{2}}{1-|z|^{2}}\right)^{\frac{q}{p}-\tau-1} \frac{(1-|\zeta|)^{\tau}}{\widehat{v}(\zeta)} \chi_{\mathbb{D} \backslash D(0,|z|)}(\zeta), \quad z \in \mathbb{D}, \quad \zeta \in D_{1}(z),
\end{aligned}
$$

because of how $\tau$ is chosen in Lemma 9. Therefore (3.8) together with Lemmas A(ii) and 3 (ii) yields

$$
\begin{aligned}
& \frac{(1-|z|)^{c+1} \gamma(z)^{q}}{\widehat{v}(z)} \int_{D_{1}(z)}\left|(1+\beta(z, \zeta)) \Gamma_{\tau}(z, \zeta)\right|^{q} \frac{\left(1-|\zeta|^{2}\right)^{\sigma}}{|1-z \bar{\zeta}|^{2+c+\sigma}} v(\zeta) d A(\zeta) \\
& \quad \lesssim \frac{(1-|z|)^{c+2+2 \tau-\delta-\frac{q}{p}} \gamma(z)^{q}}{\widehat{v}(z)^{2}} \int_{D_{1}(z) \cap D(0,|z|)} \frac{\left(1-|\zeta|^{2}\right)^{\sigma-\delta}}{|1-z \bar{\zeta}|^{4+c+\sigma-2\left(\frac{q}{p}+\delta-\tau\right)} v(\zeta) d A(\zeta)} \\
& \quad+\frac{(1-|z|)^{c+2+\tau-\delta-\frac{q}{p}} \gamma(z)^{q}}{\widehat{v}(z)} \int_{D_{1}(z) \backslash D(0,|z|)} \frac{\left(1-|\zeta|^{2}\right)^{\sigma-\delta+\tau}}{\widehat{v}(\zeta)|1-z \bar{\zeta}|^{4+c+\sigma-2\left(\frac{q}{p}+\delta-\tau\right)}} v(\zeta) d A(\zeta) \\
& \quad \frac{(1-|z|)^{\frac{c}{2}+\tau-\frac{\sigma}{2}} \gamma(z)^{q}}{\widehat{v}(z)^{2}} \int_{0}^{|z|}(1-s)^{\sigma-\delta} v(s) d s \\
& \quad+\frac{(1-|z|)^{\frac{c}{2}-\frac{\sigma}{2}} \gamma(z)^{q}}{\widehat{v}(z)} \int_{|z|}^{1}(1-s)^{\sigma-\delta+\tau} \frac{v(s)}{\widehat{v}(s)} d s \\
& \lesssim \frac{(1-|z|)^{\frac{c}{2}+\tau-\frac{\sigma}{2}+1-\frac{q}{p}}}{\widehat{v}(z) \widehat{\omega}(z)^{\frac{q}{p}}}+\frac{(1-|z|)^{\frac{c}{2}+\frac{\sigma}{2}+1+\tau-\delta-\frac{q}{p}}}{\widehat{\omega}(z)^{\frac{q}{p}}} \\
& \lesssim(1-|z|)^{\frac{c}{2}+\tau-\frac{\sigma}{2}+1-\frac{q}{p}-\beta(\nu)-\frac{q}{p} \beta(\omega)} \lesssim 1, \quad z \in \mathbb{D},
\end{aligned}
$$

where the last estimate is an immediate consequence of the choices of $c$ and $\delta$.

Case 2 If

$$
\zeta \in D_{2}(z)=\left\{w \in \mathbb{D}: 1-\frac{2|1-z \bar{w}|^{2}}{1-|z|^{2}} \geq|z| \geq|w|\right\},
$$

then $|1-z \bar{\zeta}| \asymp 1-|z|^{2} \leq 1-|\zeta|^{2}$, which together the fact that $\frac{\widehat{v}(t)}{(1-t)^{\tau}}$ and $\frac{\widehat{\omega}(r)}{(1-r)^{\tau \frac{p}{q}}}$ are essentially decreasing on $[0,1)$ gives

$$
\Gamma_{\tau}(z, \zeta)^{q} \lesssim \gamma(z)^{-q}, \quad z \in \mathbb{D}, \quad \zeta \in D_{2}(z) .
$$

Therefore (3.8) and Lemma A(iii) yield

$$
\begin{aligned}
& \frac{(1-|z|)^{c+1} \gamma(z)^{q}}{\widehat{v}(z)} \int_{D_{2}(z)}\left|(1+\beta(z, \zeta)) \Gamma_{\tau}(z, \zeta)\right|^{q} \frac{\left(1-|\zeta|^{2}\right)^{\sigma}}{|1-z \bar{\zeta}|^{2+c+\sigma}} v(\zeta) d A(\zeta) \\
& \quad \lesssim \frac{(1-|z|)^{c+1-\delta}}{\widehat{v}(z)} \int_{D_{2}(z)} \frac{\left(1-|\zeta|^{2}\right)^{\sigma-\delta}}{|1-z \bar{\zeta}|^{2+c+\sigma-2 \delta}} v(\zeta) d A(\zeta) \\
& \lesssim \frac{(1-|z|)^{c+1-\delta}}{\widehat{v}(z)} \int_{0}^{|z|} \frac{v(r)}{(1-r)^{c+1-\delta}} d r \lesssim 1, \quad z \in \mathbb{D} .
\end{aligned}
$$


Case 3 If

$$
\zeta \in D_{3}(z)=\left\{w \in \mathbb{D}: \min \left\{1-\frac{2|1-z \bar{w}|^{2}}{1-|z|^{2}},|w|\right\} \geq|z|\right\},
$$

then $|1-z \bar{\zeta}| \asymp 1-|z|^{2} \geq 1-|\zeta|^{2}$, which together the fact that $\frac{\widehat{v}(t)}{(1-t)^{\tau}}$ and $\frac{\widehat{\omega}(r)}{(1-r)^{\tau \frac{p}{q}}}$ are essentially decreasing on $[0,1)$ implies

$$
\Gamma_{\tau}(z, \zeta)^{q} \lesssim \frac{\widehat{\omega}(z)^{\frac{q}{p}}(1-|z|)^{\frac{q}{p}-1}}{\widehat{v}(\zeta)}, \quad z \in \mathbb{D}, \quad \zeta \in D_{3}(z) .
$$

Therefore (3.8) and Lemma 3(ii) imply

$$
\begin{aligned}
& \frac{(1-|z|)^{c+1} \gamma(z)^{q}}{\widehat{v}(z)} \int_{D_{3}(z)}\left|(1+\beta(z, \zeta)) \Gamma_{\tau}(z, \zeta)\right|^{q} \frac{\left(1-|\zeta|^{2}\right)^{\sigma}}{|1-z \bar{\zeta}|^{2+c+\sigma}} v(\zeta) d A(\zeta) \\
& \quad \lesssim(1-|z|)^{c+1-\delta} \int_{D_{3}(z)} \frac{\left(1-|\zeta|^{2}\right)^{\sigma-\delta}}{|1-z \bar{\zeta}|^{2+c+\sigma-2 \delta}} \frac{v(\zeta)}{\widehat{v}(\zeta)} d A(\zeta) \\
& \quad \lesssim(1-|z|)^{\delta-\sigma} \int_{|z|}^{1} \frac{(1-s)^{\sigma-\delta} v(s)}{\widehat{v}(s)} d s \lesssim 1, \quad z \in \mathbb{D} .
\end{aligned}
$$

Case 4 If

$$
\zeta \in D_{4}(z)=\left\{w \in \mathbb{D}: 1-\frac{2|1-z \bar{w}|^{2}}{1-|z|^{2}}<|z|\right\},
$$

then Lemma A(ii) gives

$$
\widehat{\omega}\left(1-\frac{2|1-z \bar{\zeta}|^{2}}{1-|z|^{2}}\right) \lesssim\left(\frac{|1-z \bar{\zeta}|}{1-|z|}\right)^{2 \beta(\omega)} \widehat{\omega}(z), \quad z \in \mathbb{D}, \quad \zeta \in D_{4}(z)
$$

and hence

$$
\begin{aligned}
\Gamma_{\tau}(z, \zeta)^{q} \lesssim & \left(\frac{|1-z \bar{\zeta}|}{1-|z|}\right)^{2 \beta(\omega) \frac{q}{p}} \widehat{\omega}(z)^{\frac{q}{p}}\left(\left(\frac{|1-z \bar{\zeta}|^{2}}{1-|\zeta|}\right)^{\frac{q}{p}-\tau-1} \frac{(1-|z|)^{\tau}}{\widehat{v}(z)} \chi_{D(0,|z|)}(\zeta)\right. \\
& \left.+\left(\frac{|1-z \bar{\zeta}|^{2}}{1-|z|}\right)^{\frac{q}{p}-\tau-1} \frac{(1-|\zeta|)^{\tau}}{\widehat{v}(\zeta)} \chi_{\mathbb{D} \backslash D(0,|z|)}(\zeta)\right), \quad z \in \mathbb{D}, \quad \zeta \in D_{4}(z)
\end{aligned}
$$

Therefore (3.8) and Lemmas A(iii) and 3 (ii) yield

$$
\begin{aligned}
& \frac{(1-|z|)^{c+1} \gamma(z)^{q}}{\widehat{v}(z)} \int_{D_{4}(z)}\left|(1+\beta(z, \zeta)) \Gamma_{\tau}(z, \zeta)\right|^{q} \frac{\left(1-|\zeta|^{2}\right)^{\sigma}}{|1-z \bar{\zeta}|^{2+c+\sigma}} v(\zeta) d A(\zeta) \\
& \quad \lesssim \frac{(1-|z|)^{c+2-\delta-\frac{q}{p}-2 \beta(\omega) \frac{q}{p}+\tau}}{\widehat{v}(z)} \int_{D_{4}(z) \cap D(0,|z|)} \frac{\left(1-|\zeta|^{2}\right)^{\sigma-\delta-\frac{q}{p}+\tau+1}}{|1-z \bar{\zeta}|^{4+c+\sigma-2 \delta-2 \beta(\omega) \frac{q}{p}-2 \frac{q}{p}+2 \tau}} v(\zeta) d A(\zeta) \\
& \quad+(1-|z|)^{c+2-\delta-\frac{q}{p}-2 \beta(\omega) \frac{q}{p}-\frac{q}{p}+\tau+1} \int_{D_{4}(z) \backslash D(0,|z|)} \frac{(1-|\zeta|)^{\sigma-\delta+\tau}}{|1-z \bar{\zeta}|^{4+c+\sigma-2 \delta-2 \beta(\omega) \frac{q}{p}-2 \frac{q}{p}+2 \tau}} \frac{v(\zeta)}{\widehat{v}(\zeta)} d A(\zeta) \\
& \lesssim \frac{(1-|z|)^{c+2-\delta-\frac{q}{p}-2 \beta(\omega) \frac{q}{p}+\tau}}{\widehat{v}(z)} \int_{0}^{|z|} \frac{v(r)}{(1-r)^{2+c-\delta-2 \beta(\omega) \frac{q}{p}-\frac{q}{p}+\tau}} d r \\
& \quad+\frac{1}{(1-|z|)^{\sigma-\delta+\tau}} \int_{|z|}^{1} \frac{(1-r)^{\sigma-\delta+\tau} v(r)}{\widehat{v}(r)} d r \lesssim 1, \quad z \in \mathbb{D} .
\end{aligned}
$$


Since $\mathbb{D}=\cup_{j=1}^{4} D_{j}(z)$ for each $z \in \mathbb{D}$, by combining the four cases we obtain (3.7). Thus (ii) implies (iii), and the proof is complete.

\section{Boundedness of integral operators}

In order to deal with the boundedness of Hankel operators, we need a technical result concerning certain integral operators. For $f \in L_{b}^{1}$ and $b, c \in \mathbb{R}$, define

$$
T_{b, c}(f)(z)=\int_{\mathbb{D}} f(\zeta) \frac{\left(1-|\zeta|^{2}\right)^{b}}{(1-z \bar{\zeta})^{c}} d A(\zeta), \quad z \in \mathbb{D},
$$

and

$$
S_{b, c}(f)(z)=\int_{\mathbb{D}} f(\zeta) \frac{\left(1-|\zeta|^{2}\right)^{b}}{|1-z \bar{\zeta}|^{c}} d A(\zeta), \quad z \in \mathbb{D} .
$$

In the analytic case the operator $T_{b, c}$ can be interpreted as a fractional differentiation or integration depending on the parameters $b$ and $c$ [20]. The boundedness of these operator between $L^{p}$ spaces induced by standard weights has been characterized in [19].

Lemma A(ii) shows that for $\eta \in \widehat{\mathcal{D}}$ there exists a constant $c_{0}=c_{0}(\sigma)>1$ such that hypotheses (i) and (ii) of the next lemma are satisfied for all $c \geq c_{0}$.

Lemma 12 Let $1<p \leq q<\infty, b>-1, c>1$ and $\sigma, \eta \in \mathcal{D}$ such that

(i) $\int_{r}^{1} \frac{(1-t)^{c-2}}{\widehat{\eta}(t)^{\frac{1}{q}}} d t \lesssim \frac{(1-r)^{c-1}}{\widehat{\eta}(r)^{\frac{1}{q}}}, \quad 0 \leq r<1$;

$$
\int_{0}^{r} \frac{\eta(t)}{(1-t)^{\frac{c q}{p}-1} \widehat{\eta}(t)^{\frac{1}{p^{\prime}}}} d t \lesssim \frac{\widehat{\eta}(r)^{\frac{1}{p}}}{(1-r)^{\frac{c q}{p}-1}}, \quad 0 \leq r<1 .
$$

Then the following statements are equivalent:

1. $S_{b, c}: A_{\sigma}^{p} \rightarrow L_{\eta}^{q}$ is bounded;

2. $T_{b, c}: A_{\sigma}^{p} \rightarrow L_{\eta}^{q}$ is bounded;

3. $\sup _{0<r<1}(1-r)^{2+b-c+\frac{1}{q}-\frac{1}{p}} \frac{\widehat{\eta}(r)^{\frac{1}{q}}}{\widehat{\sigma}(r)^{\frac{1}{p}}}<\infty$.

Proof Obviously (1) implies (2). Assume now (2), and for each $\zeta \in \mathbb{D}$ and $N \in \mathbb{N}$, define $f_{\zeta, N} \in H^{\infty}$ by $f_{\zeta, N}(z)=\frac{z^{N}}{\sigma(S(\zeta))^{\frac{1}{p}}}\left(\frac{1-|\zeta|^{2}}{1-\bar{\zeta} z}\right)^{2+b+N}$ for all $z \in \mathbb{D}$. By differentiating the reproducing formula of $A_{b}^{2}$ we obtain

$$
g^{(N)}(z)=M_{1} \int_{\mathbb{D}} \frac{\bar{u}^{N} g(u)\left(1-|u|^{2}\right)^{b}}{(1-\bar{u} z)^{2+b+N}} d A(u), \quad z \in \mathbb{D}, \quad N \in \mathbb{N}, \quad g \in A_{b}^{2},
$$

where $M_{1}=M_{1}(N, b)>0$ is a constant. Therefore

$$
\begin{aligned}
T_{b, c}\left(f_{\zeta, N}\right)(z) & =\frac{\left(1-|\zeta|^{2}\right)^{2+b+N}}{\sigma(S(\zeta))^{\frac{1}{p}}} \int_{\mathbb{D}} \frac{u^{N}\left(1-|u|^{2}\right)^{b}}{(1-u \bar{\zeta})^{2+b+N}(1-\bar{u} z)^{c}} d A(u) \\
& =\frac{\left(1-|\zeta|^{2}\right)^{2+b+N}}{\sigma(S(\zeta))^{\frac{1}{p}}} \int_{\mathbb{D}} \frac{\bar{u}^{N}\left(1-|u|^{2}\right)^{b}}{(1-\zeta \bar{u})^{2+b+N}(1-\bar{z} u)^{c}} d A(u) \\
& =M_{2} \frac{\left(1-|\zeta|^{2}\right)^{2+b+N}}{\sigma(S(\zeta))^{\frac{1}{p}}} \frac{z^{N}}{(1-z \bar{\zeta})^{c+N}},
\end{aligned}
$$


where $M_{2}=M_{2}(b, c, N)>0$. Fix $N>\max \left\{\frac{\lambda(\eta)+1}{q}-c, \frac{\lambda(\sigma)+1}{p}-b-2\right\}$. Then Lemma A(iv) gives $\left\|f_{\zeta, N}\right\|_{L_{\sigma}^{p}} \asymp 1$ and

$$
\int_{\mathbb{D}} \frac{\eta(z)}{|1-\bar{\zeta} z|^{(c+N) q}} d A(z) \asymp \frac{\eta(S(\zeta))}{(1-|\zeta|)^{(c+N) q}}, \quad \zeta \in \mathbb{D} .
$$

Therefore (2) yields

$$
\begin{aligned}
\infty & >\left\|f_{\zeta, N}\right\|_{L_{\sigma}^{p}}^{q} \gtrsim\left\|T_{b, c}\left(f_{\zeta, N}\right)\right\|_{L_{\eta}^{q}}^{q} \asymp\left(\frac{\left(1-|\zeta|^{2}\right)^{2+b+N}}{\sigma(S(\zeta))^{\frac{1}{p}}}\right)^{q} \int_{\mathbb{D}} \frac{\eta(z)}{|1-\bar{\zeta} z|^{(c+N) q}} d A(z) \\
& \asymp\left(1-|\zeta|^{2}\right)^{q(2+b-c)} \frac{\eta(S(\zeta))}{\sigma(S(\zeta))^{\frac{q}{p}}}, \quad \zeta \in \mathbb{D},
\end{aligned}
$$

thus (3) holds.

Assume (3) holds and let $h(\zeta)=\widehat{\sigma}(\zeta)^{\frac{1}{p p^{\prime}}}\left(1-|\zeta|^{2}\right)^{\frac{b}{p}+\left(\frac{1}{p}-\frac{1}{q}\right) \frac{1}{p^{\prime}}}$ for all $\zeta \in \mathbb{D}$. Then Hölder's inequality yields

$$
\begin{aligned}
& \left|S_{b, c} f(z)\right| \leq\left(\int_{\mathbb{D}}|f(\zeta)|^{p} h(\zeta)^{p} \frac{d A(\zeta)}{|1-z \bar{\zeta}|^{c}}\right)^{\frac{1}{p}}\left(\int_{\mathbb{D}}\left(\frac{\left(1-|\zeta|^{2}\right)^{b}}{h(\zeta)}\right)^{p^{\prime}} \frac{d A(\zeta)}{|1-z \bar{\zeta}|^{c}}\right)^{\frac{1}{p^{\prime}}} \\
& =I_{1}(z)^{\frac{1}{p}} \cdot I_{2}(z)^{\frac{1}{p^{\prime}}}
\end{aligned}
$$

where

$$
\begin{aligned}
I_{2}(z) & =\int_{\mathbb{D}} \frac{\left(1-|\zeta|^{2}\right)^{b-\frac{1}{p}+\frac{1}{q}}}{|1-z \bar{\zeta}|^{c} \widehat{\sigma}(\zeta)^{\frac{1}{p}}} d A(\zeta) \asymp \int_{0}^{1} \frac{(1-r)^{b-\frac{1}{p}+\frac{1}{q}}}{\widehat{\sigma}(r)^{\frac{1}{p}}(1-r|z|)^{c-1}} d r \\
& =\int_{0}^{|z|} \frac{(1-r)^{b-\frac{1}{p}+\frac{1}{q}}}{\widehat{\sigma}(r)^{\frac{1}{p}}(1-r|z|)^{c-1}} d r+\int_{|z|}^{1} \frac{(1-r)^{b-\frac{1}{p}+\frac{1}{q}}}{\widehat{\sigma}(r)^{\frac{1}{p}}(1-r|z|)^{c-1}} d r=J^{|z|}+J_{|z|} .
\end{aligned}
$$

Lemma B together with the assumption (3) yields

$$
J^{|z|} \leq \int_{0}^{|z|} \frac{(1-r)^{b-\frac{1}{p}+\frac{1}{q}+1-c}}{\widehat{\sigma}(r)^{\frac{1}{p}}} d r \lesssim \int_{0}^{|z|} \frac{d r}{\widehat{\eta}(r)^{\frac{1}{q}}(1-r)} \lesssim \frac{1}{\widehat{\eta}(z)^{\frac{1}{q}}}, \quad z \in \mathbb{D},
$$

since $\eta \in \mathcal{D} \subset \breve{\mathcal{D}}$ by the hypothesis. In a similar fashion, (3) together with the hypothesis (i) gives

$$
J_{|z|} \leq \frac{1}{(1-|z|)^{c-1}} \int_{|z|}^{1} \frac{(1-r)^{b-\frac{1}{p}+\frac{1}{q}}}{\widehat{\sigma}(r)^{\frac{1}{p}}} d r \lesssim \frac{1}{(1-|z|)^{c-1}} \int_{|z|}^{1} \frac{(1-r)^{c-2}}{\widehat{\eta}(r)^{\frac{1}{q}}} d r \lesssim \frac{1}{\widehat{\eta}(z)^{\frac{1}{q}}}, \quad z \in \mathbb{D},
$$

and hence $I_{2}(z) \lesssim \widehat{\eta}(z)^{-\frac{1}{q}}$ for all $z \in \mathbb{D}$. This estimate and Minkowski's integral inequality (Fubini's theorem in the case $q=p$ ) now yield

$$
\begin{aligned}
\left\|S_{b, c}(f)\right\|_{L_{\eta}^{q}}^{p} & \lesssim\left(\int_{\mathbb{D}}\left(\int_{\mathbb{D}}|f(\zeta)|^{p} h(\zeta)^{p} \frac{d A(\zeta)}{|1-z \bar{\zeta}|^{c}}\right)^{\frac{q}{p}} \frac{\eta(z)}{\widehat{\eta}(z)^{\frac{1}{p^{\prime}}}} d A(z)\right)^{\frac{p}{q}} \\
& \leq \int_{\mathbb{D}}|f(\zeta)|^{p} \widetilde{\sigma}(\zeta) I_{3}(\zeta) d A(\zeta),
\end{aligned}
$$


where

$$
I_{3}(\zeta)=\frac{h(\zeta)^{p}}{\widetilde{\sigma}(\zeta)}\left(\int_{\mathbb{D}} \frac{\eta(z) d A(z)}{|1-z \bar{\zeta}|^{\frac{c q}{p}} \widehat{\eta}(z)^{\frac{1}{p^{\prime}}}}\right)^{\frac{p}{q}} \asymp \frac{h(\zeta)^{p}}{\widetilde{\sigma}(\zeta)}\left(\int_{0}^{1} \frac{\eta(r)}{(1-r|\zeta|)^{\frac{c q}{p}}-1 \hat{\eta}(r)^{\frac{1}{p^{\prime}}}} d r\right)^{\frac{p}{q}}
$$

Since

$$
\int_{0}^{|\zeta|} \frac{\eta(r)}{(1-r|\zeta|)^{\frac{c q}{p}-1} \widehat{\eta}(r)^{1 / p^{\prime}}} d r \leq \int_{0}^{|\zeta|} \frac{\eta(r)}{(1-r)^{\frac{c q}{p}-1} \widehat{\eta}(r)^{\frac{1}{p^{\prime}}}} d r \lesssim \frac{\widehat{\eta}(\zeta)^{\frac{1}{p}}}{(1-|\zeta|)^{\frac{c q}{p}-1}}, \quad \zeta \in \mathbb{D},
$$

by the hypothesis (ii), and

$$
\int_{|\zeta|}^{1} \frac{\eta(r)}{(1-r|\zeta|)^{\frac{c q}{p}-1} \widehat{\eta}(r)^{\frac{1}{p^{\prime}}}} d r \leq \frac{1}{(1-|\zeta|)^{\frac{c q}{p}-1}} \int_{|\zeta|}^{1} \frac{\eta(r)}{\widehat{\eta}(r)^{\frac{1}{p^{\prime}}}} d r \asymp \frac{\widehat{\eta}(\zeta)^{\frac{1}{p}}}{(1-|\zeta|)^{\frac{c q}{p}-1}}, \quad \zeta \in \mathbb{D},
$$

we deduce

$$
I_{3}(\zeta) \lesssim(1-|\zeta|)^{2+b-c+\frac{1}{q}-\frac{1}{p}} \frac{\widehat{\eta}(\zeta)^{\frac{1}{q}}}{\widehat{\sigma}(\zeta)^{\frac{1}{p}}} \lesssim 1, \quad \zeta \in \mathbb{D}
$$

by the assumption (3). It follows that $\left\|S_{b, c}(f)\right\|_{L_{\eta}^{q}} \lesssim\|f\|_{A_{\tilde{\sigma}}^{p}}$. This finishes the proof because $\|f\|_{A_{\tilde{\sigma}}^{p}} \asymp\|f\|_{A_{\sigma}^{p}}$ for all $f \in \mathcal{H}(\mathbb{D})$ by Lemma 5 provided $\sigma \in \mathcal{D}$.

\section{Proof of Theorem 1}

In order to prove the sufficiency part of Theorem 1 we shall use the next result which follows from the argument used in the proof of [12, Lemma 4.5].

Lemma 13 Let $1<q<\infty$ and $v$, $\omega$ weights such that $P_{\omega}: L_{v}^{q} \rightarrow L_{v}^{q}$ is bounded. Then

$$
\left\|H_{f}^{v}(g)\right\|_{L_{v}^{q}}^{q} \leq\left(1+\left\|P_{\omega}\right\|_{L_{v}^{q} \rightarrow L_{v}^{q}}\right)\left\|H_{f}^{\omega}(g)\right\|_{L_{v}^{q}}^{q}, \quad f \in L_{v}^{q}, \quad g \in H^{\infty} .
$$

Proposition 14 Let $1<p \leq q<\infty, v \in B_{q}$ a radial weight and $\omega \in \mathcal{D}$. If $f \in \mathrm{BO}(\Delta)$, then $H_{f}^{v}: A_{\omega}^{p} \rightarrow L_{v}^{q}$ is bounded.

Proof By [5] there exists a constant $s_{0}=s_{0}(v)>-1$ such that $P_{s}: L_{v}^{q} \rightarrow L_{v}^{q}$ is bounded for each $s>s_{0}$. Let $0<\tau<\min \{q \alpha(\omega) / p, \alpha(v)\}$, where $\alpha(v)$ and $\alpha(\omega)$ are those from Lemma B. Then Lemmas 9 and 13 yield

$$
\begin{aligned}
\left\|H_{f}^{v}(g)\right\|_{L_{v}^{q}}^{q} & \lesssim\left\|H_{f}^{s}(g)\right\|_{L_{v}^{q}}^{q} \leq \int_{\mathbb{D}}\left(\int_{\mathbb{D}} \frac{|f(z)-f(\zeta)||g(\zeta)|}{|1-\bar{z} \zeta|^{2+s}}\left(1-|\zeta|^{2}\right)^{s} d A(\zeta)\right)^{q} v(z) d A(z) \\
& \lesssim \int_{\mathbb{D}}\left(\int_{\mathbb{D}}|g(\zeta)| \frac{(\beta(z, \zeta)+1) \Gamma_{\tau}(z, \zeta)}{|1-\bar{z} \zeta|^{2+s}}\left(1-|\zeta|^{2}\right)^{s} d A(\zeta)\right)^{q} v(z) d A(z), \quad g \in H^{\infty} .
\end{aligned}
$$

Let $s>\max \left\{s_{0}, 2(\beta(\omega)+\beta(v)+2 \alpha(v))\right\}, \delta<\min \left\{\frac{\tau}{q}, \frac{\alpha(v)}{q}\right\}$ and $K>1$ to be fixed later. Then applying (3.8), we get

$$
\begin{aligned}
\left\|H_{f}^{v}(g)\right\|_{L_{v}^{q}}^{q} & \lesssim \sum_{j=1}^{5} \int_{\mathbb{D}}\left(\int_{\Omega_{j}(z)}|g(\zeta)| \frac{\Gamma_{\tau}(z, \zeta) d A(\zeta)}{|1-\bar{z} \zeta|^{2+s-2 \delta}\left(1-|\zeta|^{2}\right)^{\delta-s}}\right)^{q} \frac{v(z)}{(1-|z|)^{q \delta}} d A(z) \\
& =\sum_{j=1}^{5} I_{j}(g),
\end{aligned}
$$


where

$$
\begin{aligned}
\Omega_{1}(z) & =\left\{\zeta \in \mathbb{D}: \frac{1}{|1-\bar{z} \zeta|^{2}} \leq \frac{2}{\max \left\{1-|z|^{2}, 1-|\zeta|^{2}\right\}}\right\} \cap D(0,|z|), \\
\Omega_{2}(z) & =\left\{\zeta \in \mathbb{D}: \frac{1}{|1-\bar{z} \zeta|^{2}} \leq \frac{2}{\max \left\{1-|z|^{2}, 1-|\zeta|^{2}\right\}}\right\} \cap(\mathbb{D} \backslash D(0,|z|)), \\
\Omega_{3}(z, K) & =\left\{\zeta \in \mathbb{D}: \frac{1-|\zeta|}{K} \geq \frac{2|1-\bar{z} \zeta|^{2}}{\max \left\{1-|z|^{2}, 1-|\zeta|^{2}\right\}}\right\}, \\
\Omega_{4}(z, K) & =\left\{\zeta \in \mathbb{D}: \frac{1-|\zeta|}{K}<\frac{2|1-\bar{z} \zeta|^{2}}{\max \left\{1-|z|^{2}, 1-|\zeta|^{2}\right\}}<1\right\} \cap D(0,|z|), \\
\Omega_{5}(z, K) & =\left\{\zeta \in \mathbb{D}: \frac{1-|\zeta|}{K}<\frac{2|1-\bar{z} \zeta|^{2}}{\max \left\{1-|z|^{2}, 1-|\zeta|^{2}\right\}}<1\right\} \cap(\mathbb{D} \backslash D(0,|z|)) .
\end{aligned}
$$

The quantities $I_{j}(g), j=1, \ldots, 5$, will be estimated separately.

Case $I_{1}(g)$ By using the definition of $\Omega_{1}(z)$, and the fact that $\frac{\widehat{v}(x)}{(1-x)^{\tau}}$ is essentially decreasing on $[0,1)$ we deduce

$$
\Gamma_{\tau}(z, \zeta) \lesssim \frac{\left(\frac{|1-\bar{z} \zeta|^{2}}{\max \left\{1-|z|^{2}, 1-|\zeta|^{2}\right\}}\right)^{\frac{1}{p}-\frac{1}{q}}}{\min \left\{\frac{\widehat{v}(z)}{(1-|z|)^{\tau}}, \frac{\widehat{v}(\zeta)}{(1-|\zeta|)^{\tau}}\right\}^{\frac{1}{q}}} \lesssim\left(\frac{|1-\bar{z} \zeta|^{2}}{1-|\zeta|^{2}}\right)^{\frac{1}{p}-\frac{1}{q}}\left(\frac{(1-|z|)^{\tau}}{\widehat{v}(z)}\right)^{\frac{1}{q}}, \quad z \in \mathbb{D}, \quad \zeta \in \Omega_{1}(z)
$$

Then the estimate

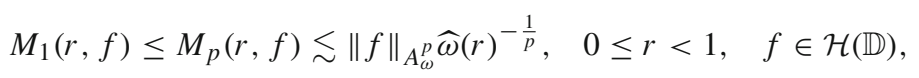

and Lemma 3(ii) yield

$$
\begin{aligned}
& I_{1}(g) \lesssim \int_{\mathbb{D}}\left(\int_{\Omega_{1}(z)}|g(\zeta)| \frac{(1-|\zeta|)^{s-\delta-\frac{1}{p}+\frac{1}{q}}}{|1-\bar{z} \zeta|^{2+s-2 \delta-2\left(\frac{1}{p}-\frac{1}{q}\right)}} d A(\zeta)\right)^{q} \frac{v(z)(1-|z|)^{\tau-\delta q}}{\widehat{v}(z)} d A(z) \\
& \lesssim\left(\int_{\mathbb{D}}|g(\zeta)|(1-|\zeta|)^{\frac{s}{2}-1} d A(\zeta)\right)^{q} \int_{\mathbb{D}} \frac{v(z)(1-|z|)^{\tau-\delta q}}{\widehat{v}(z)} d A(z) \\
& \lesssim\|g\|_{A_{\omega}^{p}}^{q}\left(\int_{0}^{1} \frac{(1-t)^{\frac{s}{2}-1}}{\widehat{\omega}(t)^{\frac{1}{p}}} d t\right)^{q} \lesssim\|g\|_{A_{\omega}^{p}}^{q}\left(\int_{0}^{1}(1-t)^{\frac{s}{2}-1-\frac{\beta(\omega)}{p}} d t\right)^{q} \lesssim\|g\|_{A_{\omega}^{p}}^{q}, \quad g \in H^{\infty} .
\end{aligned}
$$

Case $I_{2}(g)$ The definition of $\Omega_{2}(z)$ and the fact that $\frac{\widehat{v}(x)}{(1-x)^{\tau}}$ is essentially decreasing imply

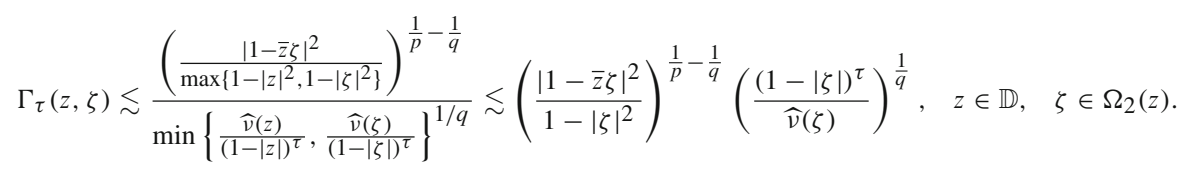


Therefore (5.2) and Lemmas A and B yield

$$
\begin{aligned}
I_{2}(g) & \lesssim \int_{\mathbb{D}}\left(\int_{\Omega_{2}(z)}|g(\zeta)| \frac{(1-|\zeta|)^{s-\delta-\frac{1}{p}+\frac{1+\tau}{q}}}{\widehat{v}(\zeta)^{\frac{1}{q}}|1-\bar{z} \zeta|^{2+s-2 \delta-2\left(\frac{1}{p}-\frac{1}{q}\right)}} d A(\zeta)\right)^{q}(1-|z|)^{-\delta q} v(z) d A(z) \\
& \lesssim\left(\int_{\mathbb{D}}|g(\zeta)| \frac{(1-|\zeta|)^{\frac{s}{2}-1+\frac{\tau}{q}}}{\widehat{v}(\zeta)^{\frac{1}{q}}} d A(\zeta)\right)^{q} \int_{0}^{1}(1-r)^{-\delta q} \nu(r) d r \\
& \lesssim\|g\|_{A_{\omega}^{p}}^{q}\left(\int_{0}^{1} \frac{(1-r)^{\frac{s}{2}-1+\frac{\tau}{q}}}{\widehat{\omega}(r)^{\frac{1}{p}} \widehat{v}(\zeta)^{\frac{1}{q}}} d A(\zeta)\right)^{q}\left(\widehat{v}(0)+\int_{0}^{1} \frac{\widehat{v}(t)}{(1-t)^{1+q \delta}} d t\right) \\
& \lesssim\|g\|_{A_{\omega}^{p}}^{q}\left(\int_{0}^{1}(1-t)^{\frac{s}{2}-1-\frac{\beta(\omega)}{p}-\frac{\beta(v)-\tau}{q}} d t\right)^{q} \lesssim\|g\|_{A_{\omega}^{p},}^{q}, g \in H^{\infty} .
\end{aligned}
$$

Case $I_{3}(g)$ To deal with $I_{3}(g)$, note first that now $2 K|1-\bar{z} \zeta|^{2} \leq(1-|\zeta|) \max \{1-$ $\left.|z|^{2}, 1-|\zeta|^{2}\right\} \leq 2(\max \{1-|z|, 1-|\zeta|\})^{2}$ for all $\zeta \in \Omega_{3}(z, K)$. Hence $\zeta \in \Delta(z, R)$ for some $R=R(K) \in(0, \infty)$ if $K \geq 1$ is sufficiently large. Fix such a $K$, and note that then $\widehat{v}(\zeta) \asymp \widehat{v}(z)$ for all $\zeta \in \Omega(z, K)$ by Lemma A(ii). By using this and the fact that $\frac{\widehat{\omega}(x)}{(1-x)^{\frac{p \tau}{q}}}$ is essentially decreasing on $[0,1)$ we deduce

$$
\begin{aligned}
\Gamma_{\tau}(z, \zeta) & \lesssim\left(\frac{|1-\bar{z} \zeta|^{2}}{\max \left\{1-|z|^{2}, 1-|\zeta|^{2}\right\}}\right)^{\frac{1}{p}-\frac{1}{q}} \frac{\widehat{\omega}(\zeta)^{\frac{1}{p}}}{(1-|\zeta|)^{\frac{\tau}{q}}} \min \left\{\frac{\widehat{v}(z)}{(1-|z|)^{\tau}}, \frac{\widehat{v}(\zeta)}{(1-|\zeta|)^{\tau}}\right\}^{-\frac{1}{q}} \\
& \asymp \frac{(1-|\zeta|)^{\frac{1}{p}-\frac{1}{q}} \widehat{\omega}(\zeta)^{\frac{1}{p}}}{\widehat{v}(\zeta)^{\frac{1}{q}}}, \quad z \in \mathbb{D}, \quad \zeta \in \Omega_{3}(z, K),
\end{aligned}
$$

and it follows that

$$
\begin{aligned}
I_{3}(g) & \lesssim \int_{\mathbb{D}}\left(\int_{\Delta(z, R)}\left(|g(\zeta)| \widehat{\omega}(\zeta)^{\frac{1}{p}}\right) \frac{\left(1-|\zeta|^{2}\right)^{s-\delta+\frac{1}{p}-\frac{2}{q}} d A(\zeta)}{|1-\bar{z} \zeta|^{2+s-2 \delta}}\right)^{q} \frac{v(z)(1-|z|)^{1-q \delta}}{\widehat{v}(z)} d A(z) \\
& \asymp \int_{\mathbb{D}}\left(\int_{\Delta(z, R)}\left(|g(\zeta)| \widetilde{\omega}(\zeta)^{\frac{1}{p}}\right) \frac{\left(1-|\zeta|^{2}\right)^{s+\frac{2}{p}-\frac{2}{q}} d A(\zeta)}{|1-\bar{z} \zeta|^{2+s}}\right)^{q} \frac{v(z)(1-|z|)}{\widehat{v}(z)} d A(z) \\
& \asymp \int_{\mathbb{D}}\left(\int_{\Delta(z, R)}\left(|g(\zeta)| \widetilde{\omega}(\zeta)^{\frac{1}{p}}\right) \frac{\left(1-|\zeta|^{2}\right)^{s} d A(\zeta)}{|1-\bar{z} \zeta|^{2+s-\frac{2}{p}+\frac{2}{q}}}\right)^{q} \frac{v(z)(1-|z|)}{\widehat{v}(z)} d A(z) \\
& \leq \int_{\mathbb{D}}\left(\int_{\mathbb{D}}\left(|g(\zeta)| \widetilde{\omega}(\zeta)^{\frac{1}{p}}\right) \frac{\left(1-|\zeta|^{2}\right)^{s} d A(\zeta)}{\left.|1-\bar{z} \zeta|^{2+s-\frac{2}{p}+\frac{2}{q}}\right)^{q} \frac{v(z)(1-|z|)}{\widehat{v}(z)} d A(z)}\right. \\
& =\left\|S_{s, s+2\left(1-\frac{1}{p}+\frac{1}{q}\right)}\left(|g| \widetilde{\omega}^{\frac{1}{p}}\right)\right\|_{L_{\eta}^{q}}^{q}=\left\|S_{b, c}\left(|g| \widetilde{\omega}^{\frac{1}{p}}\right)\right\|_{L_{\eta}^{q}}^{q}, g \in H^{\infty},
\end{aligned}
$$

where $\eta(z)=\frac{v(z)(1-|z|)}{\widehat{v}(z)}$ for all $z \in \mathbb{D}$. To apply Lemma 12 with $\sigma \equiv 1$, we must check that its hypotheses are satisfied. To do this, first observe that $\eta \in \mathcal{D}$ and $\widehat{\eta}(r) \asymp(1-r)$ for all 
$0 \leq r<1$ by Lemma 4 . Hence

$$
\int_{r}^{1} \frac{(1-t)^{c-2}}{\widehat{\eta}(t)^{\frac{1}{q}}} d t \asymp \int_{r}^{1}(1-t)^{s-\frac{2}{p}+\frac{1}{q}} d t \asymp(1-r)^{1+s-\frac{2}{p}+\frac{1}{q}} \asymp \frac{(1-r)^{c-1}}{\widehat{\eta}(r)^{\frac{1}{q}}}, \quad 0 \leq r<1,
$$

and, by Lemma 3(iii),

$$
\begin{aligned}
\int_{0}^{r} \frac{\eta(t)}{(1-t)^{\frac{c q}{p}-1} \widehat{\eta}(t)^{\frac{1}{p^{\prime}}}} d t & \asymp \int_{0}^{r} \frac{v(t)}{\widehat{v}(t)(1-t)^{\frac{q}{p}\left(s+2\left(1-\frac{1}{p}+\frac{1}{q}\right)\right)-1-\frac{1}{p}}} d t \\
& \lesssim \frac{1}{(1-r)^{\frac{q}{p}\left(s+2\left(1-\frac{1}{p}+\frac{1}{q}\right)\right)-1-\frac{1}{p}}} \asymp \frac{\widehat{\eta}(r)^{\frac{1}{p}}}{(1-r)^{\frac{c q}{p}-1}}, \quad 0 \leq r<1,
\end{aligned}
$$

so the hypotheses of Lemma 12 are satisfied. Moreover,

$$
(1-r)^{2+b-c+\frac{1}{q}-\frac{1}{p}} \frac{\widehat{\eta}(r)^{\frac{1}{q}}}{\widehat{\sigma}(r)^{\frac{1}{p}}} \asymp 1, \quad 0 \leq r<1,
$$

and consequently (5.3) and Lemmas 12 and 5 yield $I_{3}(g) \lesssim\|g\|_{A_{\widetilde{\omega}}^{p}}^{q} \asymp\|g\|_{A_{\omega}^{p}}^{q}$ for all $g \in H^{\infty}$.

Case $I_{4}(g)$ By using the definition of $\Omega_{4}(z, K)$, Lemma A(ii) and the fact that $\frac{\widehat{v}(x)}{(1-x)^{\tau}}$ is essentially decreasing on $[0,1)$, we deduce

$$
\begin{aligned}
& \Gamma_{\tau}(z, \zeta) \lesssim \frac{\left(\frac{|1-\bar{z} \zeta|^{2}}{\max \left\{1-|z|^{2}, 1-|\zeta|^{2}\right\}}\right)^{\frac{1}{p}-\frac{1}{q}} \widehat{\omega}\left(1-\frac{2|1-\bar{z} \zeta|^{2}}{\max \left\{1-|z|^{2}, 1-|\zeta|^{2}\right\}}\right)^{\frac{1}{p}}}{(1-|\zeta|)^{\frac{\tau}{q}} \min \left\{\frac{\widehat{v}(z)}{(1-|z|)^{\tau}}, \frac{\widehat{v}(\zeta)}{(1-|\zeta|)^{\tau}}\right\}^{\frac{1}{q}}} \\
& \lesssim \frac{\left(\frac{|1-\bar{z} \zeta|^{2}}{1-|\zeta|^{\frac{1}{p}-\frac{1}{q}}} \widehat{\omega}\left(1-\frac{K 2|1-\bar{z} \zeta|^{2}}{\max \left\{1-|z|^{2}, 1-|\zeta|^{2}\right\}}\right)^{\frac{1}{p}}\right.}{(1-|\zeta|)^{\frac{\tau}{q}} \min \left\{\frac{\widehat{v}(z)}{(1-|z|)^{\tau}}, \frac{\widehat{v}(\zeta)}{(1-|\zeta|)^{\tau}}\right\}^{\frac{1}{q}}} \lesssim \frac{\left(\frac{|1-\bar{z} \zeta|^{2}}{1-|\zeta|}\right)^{\frac{1}{p}-\frac{1}{q}}\left(\frac{|1-\bar{z} \zeta|}{1-|\zeta|}\right)^{\frac{2 \beta(\omega)}{p}} \widehat{\omega}(\zeta)^{\frac{1}{p}}}{(1-|\zeta|)^{\frac{\tau}{q}} \min \left\{\frac{\widehat{v}(z)}{(1-|z|)^{\tau}}, \frac{\widehat{v}(\zeta)}{(1-|\zeta|)^{\tau}}\right\}^{\frac{1}{q}}} \\
& \lesssim \frac{|1-\bar{z} \zeta|^{\frac{2 \beta(\omega)}{p}+\frac{2}{p}-\frac{2}{q}}}{(1-|\zeta|)^{\frac{2 \beta(\omega)}{p}+\frac{1}{p}-\frac{1}{q}}} \frac{\widehat{\omega}(\zeta)^{\frac{1}{p}}}{(1-|\zeta|)^{\frac{\tau}{q}}}\left(\frac{(1-|z|)^{\tau}}{\widehat{v}(z)}\right)^{\frac{1}{q}}, \quad z \in \mathbb{D}, \quad \zeta \in \Omega_{4}(z, K) .
\end{aligned}
$$

Therefore

$$
\begin{aligned}
& I_{4}(g) \lesssim \int_{\mathbb{D}}\left(\int_{\mathbb{D}}\left(|g(\zeta)| \widetilde{\omega}(\zeta)^{\frac{1}{p}}\right) \frac{(1-|\zeta|)^{s-\delta-\frac{2 \beta(\omega)}{p}+\frac{1}{q}-\frac{\tau}{q}}}{|1-\bar{z} \zeta|^{2+s-2 \delta-\frac{2 \beta(\omega)}{p}-\frac{2}{p}+\frac{2}{q}}} d A(\zeta)\right)^{q} \frac{v(z)(1-|z|)^{\tau-\delta q}}{\widehat{v}(z)} d A(z) \\
& =\left\|S_{b, c}\left(|g| \tilde{\omega}^{\frac{1}{p}}\right)\right\|_{L_{\eta}^{q}}^{q}, \quad g \in H^{\infty},
\end{aligned}
$$

where $b=s-\delta-\frac{2 \beta(\omega)}{p}+\frac{1}{q}-\frac{\tau}{q}, c=2+s-2 \delta-\frac{2 \beta(\omega)}{p}-\frac{2}{p}+\frac{2}{q}$ and $\eta(z)=\frac{v(z)(1-|z|)^{\tau-\delta q}}{\widehat{v}(z)}$ for all $z \in \mathbb{D}$. We will appeal to Lemma 12 with $\sigma \equiv 1$. First observe that $\eta \in \mathcal{D}$ and $\widehat{\eta}(r) \asymp(1-r)^{\tau-\delta q}$ for all $0 \leq r<1$ by Lemma 4 . Hence

$$
\begin{aligned}
\int_{r}^{1} \frac{(1-t)^{c-2}}{\widehat{\eta}(t)^{\frac{1}{q}}} d t & \asymp \int_{r}^{1}(1-t)^{s-\delta-\frac{2 \beta(\omega)}{p}-\frac{\tau}{q}-\frac{2}{p}+\frac{2}{q}} d t \asymp(1-r)^{1+s-\delta-\frac{2 \beta(\omega)}{p}-\frac{\tau}{q}-\frac{2}{p}+\frac{2}{q}} \\
& \asymp \frac{(1-r)^{c-1}}{\widehat{\eta}(r)^{\frac{1}{q}}}, \quad 0 \leq r<1,
\end{aligned}
$$


and, by Lemma 3(iii),

$$
\begin{aligned}
& \int_{0}^{r} \frac{\eta(t)}{(1-t)^{\frac{c q}{p}-1} \widehat{\eta}(t)^{\frac{1}{p^{\prime}}}} d t \asymp \int_{0}^{r} \frac{v(t)}{\widehat{v}(t)(1-t)^{\frac{q}{p}\left(2+s-2 \delta-\frac{2 \beta(\omega)}{p}-\frac{2}{p}+\frac{2}{q}\right)-1-\frac{\tau-q \delta}{p}}} d t
\end{aligned}
$$

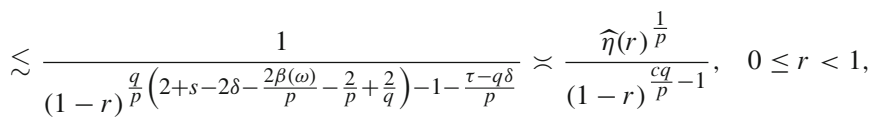

so the hypotheses of Lemma 12 are satisfied. Moreover,

$$
(1-r)^{2+b-c+\frac{1}{q}-\frac{1}{p}} \frac{\widehat{\eta}(r)^{\frac{1}{q}}}{\widehat{\sigma}(r)^{\frac{1}{p}}} \asymp 1, \quad 0 \leq r<1,
$$

and hence (5.4) and Lemmas 12 and 5 imply $I_{4}(g) \lesssim\|g\|_{A_{\widetilde{\omega}}^{p}}^{q} \asymp\|g\|_{A_{\omega}^{p}}^{q}$ for all $g \in H^{\infty}$.

Case $I_{5}(g)$ By using the definition of $\Omega_{5}(z, K)$, Lemma A(ii) and the fact that $\frac{\widehat{v}(x)}{(1-x)^{\tau}}$ is essentially decreasing on $[0,1)$ we deduce

$$
\begin{aligned}
\Gamma_{\tau}(z, \zeta) & \lesssim \frac{\left(\frac{|1-\bar{z} \zeta|^{2}}{\max \left\{1-|z|^{2}, 1-|\zeta|^{2}\right\}}\right)^{\frac{1}{p}-\frac{1}{q}} \widehat{\omega}\left(1-\frac{2|1-\bar{z} \zeta|^{2}}{\max \left\{1-|z|^{2}, 1-|\zeta|^{2}\right\}}\right)^{\frac{1}{p}}}{(1-|\zeta|)^{\frac{\tau}{q}} \min \left\{\frac{\widehat{v}(z)}{(1-|z|)^{\tau}}, \frac{\widehat{v}(\zeta)}{(1-|\zeta|)^{\tau}}\right\}^{\frac{1}{q}}} \\
& \lesssim \frac{\left(\frac{|1-\bar{z} \zeta|^{2}}{1-|\zeta|}\right)^{\frac{1}{p}-\frac{1}{q}} \widehat{\omega}\left(1-\frac{K 2|1-\bar{z} \zeta|^{2}}{\max \left\{1-|z|^{2}, 1-|\zeta|^{2}\right\}}\right)^{\frac{1}{p}}}{(1-|\zeta|)^{\frac{\tau}{q}} \min \left\{\frac{\widehat{v}(z)}{(1-|z|)^{\tau}}, \frac{\widehat{v}(\zeta)}{(1-|\zeta|)^{\tau}}\right\}^{\frac{1}{q}}} \underset{\left(\frac{|1-\bar{z} \zeta|^{2}}{1-|\zeta|}\right)^{\frac{1}{p}-\frac{1}{q}}\left(\frac{|1-\bar{z} \zeta|}{1-|\zeta|^{2}}\right)^{\frac{2 \beta(\omega)}{p}} \widehat{\omega}(\zeta)^{\frac{1}{p}}}{(1-|\zeta|)^{\frac{\tau}{q}} \min \left\{\frac{\widehat{v}(z)}{(1-|z|)^{\tau}}, \frac{\widehat{v}(\zeta)}{(1-|\zeta|)^{\tau}}\right\}^{\frac{1}{q}}} \\
& \lesssim\left(\frac{|1-\bar{z} \zeta|^{2}}{1-|\zeta|}\right)^{\frac{1}{p}-\frac{1}{q}}\left(\frac{|1-\bar{z} \zeta|}{1-|\zeta|}\right)^{\frac{2 \beta(\omega)}{p}} \widehat{\widehat{\omega}(\zeta)^{\frac{1}{p}}} \frac{\hat{v}(\zeta)^{\frac{1}{q}}}{}, \quad z \in \mathbb{D}, \quad \zeta \in \Omega_{5}(z, K) .
\end{aligned}
$$

Therefore Lemma A(ii) yields

$$
\begin{aligned}
& I_{5}(g) \lesssim \int_{\mathbb{D}}\left(\int_{\Omega_{5}(z, K)}\left(|g(\zeta)| \widetilde{\omega}(\zeta)^{\frac{1}{p}}\right) \frac{(1-|\zeta|)^{s-\delta-\frac{2 \beta(\omega)}{p}+\frac{1}{q}}}{\widehat{v}(\zeta)^{\frac{1}{q}}|1-\bar{z} \zeta|^{2+s-2 \delta-\frac{2 \beta(\omega)}{p}-\frac{2}{p}+\frac{2}{q}}} d A(\zeta)\right)^{q} \frac{\nu(z) d A(z)}{(1-|z|)^{q \delta}} \\
& \lesssim \int_{\mathbb{D}}\left(\int_{\mathbb{D}}\left(|g(\zeta)| \widetilde{\omega}(\zeta)^{\frac{1}{p}}\right) \frac{(1-|\zeta|)^{s-\delta-\frac{2 \beta(\omega)}{p}+\frac{1}{q}-\frac{\beta(\nu)}{q}}}{|1-\bar{z} \zeta|^{2+s-2 \delta-\frac{2 \beta(\omega)}{p}-\frac{2}{p}+\frac{2}{q}}} d A(\zeta)\right)^{q} \frac{v(z) d A(z)}{(1-|z|)^{q \delta-\beta(v)} \widehat{v}(z)^{\frac{1}{q}}} \\
& =\left\|S_{b, c}\left(|g| \tilde{\omega}^{\frac{1}{p}}\right)\right\|_{L_{\eta}^{q}}^{q}, \quad g \in H^{\infty},
\end{aligned}
$$

where $b=s-\delta-\frac{2 \beta(\omega)}{p}+\frac{1}{q}-\frac{\beta(\nu)}{q}, c=2+s-2 \delta-\frac{2 \beta(\omega)}{p}-\frac{2}{p}+\frac{2}{q}$ and $\eta(z)=\frac{\nu(z)(1-|z|)^{\beta(v)-\delta q}}{\widehat{v}(z)}$ for all $z \in \mathbb{D}$. Again we will appeal to Lemma 12 with $\sigma \equiv 1$. First observe that $\eta \in \mathcal{D}$ and $\widehat{\eta}(r) \asymp(1-r)^{\beta(v)-\delta q}$ for all $0 \leq r<1$ by Lemma 4 . Hence

$$
\begin{aligned}
\int_{r}^{1} \frac{(1-t)^{c-2}}{\widehat{\eta}(t)^{\frac{1}{q}}} d t & \asymp \int_{r}^{1}(1-t)^{s-\delta+\frac{2 \beta(\omega)}{p}-\frac{2}{p}+\frac{2}{q}-\frac{\beta(\nu)}{q}} d t \asymp(1-r)^{1+s-\delta+\frac{2 \beta(\omega)}{p}-\frac{2}{p}+\frac{2}{q}-\frac{\beta(\nu)}{q}} \\
& \asymp \frac{(1-r)^{c-1}}{\widehat{\eta}(r)^{\frac{1}{q}}}, \quad 0 \leq r<1,
\end{aligned}
$$


and, by Lemma 3(iii),

$$
\begin{aligned}
& \int_{0}^{r} \frac{\eta(t)}{(1-t)^{\frac{c q}{p}-1} \widehat{\eta}(t)^{1 / p^{\prime}}} d t \asymp \int_{0}^{r} \frac{v(t)}{\widehat{v}(t)(1-t)^{\frac{q}{p}\left(2+s-2 \delta-\frac{2 \beta(\omega)}{p}-\frac{2}{p}+\frac{2}{q}\right)-1-\frac{\beta(v)-q \delta}{p}}} d t
\end{aligned}
$$

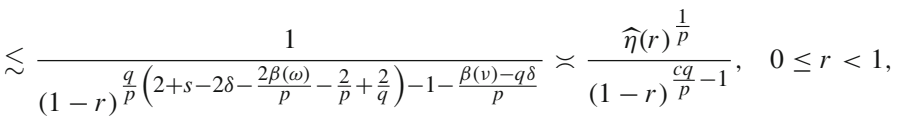

so the hypotheses of Lemma 12 are satisfied. Moreover,

$$
(1-r)^{2+b-c+\frac{1}{q}-\frac{1}{p}} \frac{\widehat{\eta}(r)^{\frac{1}{q}}}{\widehat{\sigma}(r)^{\frac{1}{p}}} \asymp 1, \quad 0 \leq r<1,
$$

and hence (5.5) together with Lemmas 5 and 12 imply $I_{5}(g) \lesssim\|g\|_{A_{\widetilde{\omega}}^{p}}^{q} \asymp\|g\|_{A_{\omega}^{p}}^{q}$ for all $g \in H^{\infty}$. This finishes the proof of the proposition.

In order to prove the necessity part of Theorem 1 some definitions are needed. For $\eta>-1$ and a radial weight $\omega$, let $b_{z, \omega}^{\eta}=B_{z}^{\eta} /\left\|B_{z}^{\eta}\right\|_{A_{\omega}^{p}}$ for $z \in \mathbb{D}$, where $B_{z}^{\eta}(\zeta)=(1-\bar{z} \zeta)^{-(2+\eta)}$. For each $f \in L_{v}^{1}$, define

$$
g_{z, \omega}^{\eta}(\zeta)=\frac{P_{v}\left(\bar{f} b_{z, \omega}^{\eta}\right)(\zeta)}{b_{z, \omega}^{\eta}(\zeta)}, \quad \zeta \in \mathbb{D}
$$

and note that $g_{z, \omega}^{\eta}$ is a well-defined analytic function in $\mathbb{D}$ because the standard Bergman kernel $b_{z, \omega}^{\eta}$ has no zeros. If $v, \omega$ are weights, $\eta>-1$ and $0<p, q<\infty$, let us consider the global mean oscillation

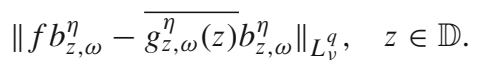

Proposition 15 Let $1<p \leq q<\infty, f \in L_{v}^{q}, \omega \in \widehat{\mathcal{D}}, v \in B_{q}$ a radial weight and $\gamma(z)=\gamma_{\omega, \nu, p, q}(z)=\frac{\widehat{v}(z)^{\frac{1}{q}}(1-|z|)^{\frac{1}{q}}}{\widehat{\omega}(z)^{\frac{1}{p}}(1-|z|)^{\frac{1}{p}}}$. If $H_{f}^{\nu}, H_{\frac{v}{f}}: A_{\omega}^{p} \rightarrow L_{\nu}^{q}$ are bounded, then there exists $\eta_{0}=\eta_{0}(v, \omega)>-1$ such that

$$
\sup _{z \in \mathbb{D}}\left\|f b_{z, \omega}^{\eta}-\overline{g_{z, \omega}^{\eta}(z)} b_{z, \omega}^{\eta}\right\|_{L_{v}^{q}} \leq\left\|H_{f}^{v}\right\|_{A_{\omega}^{p} \rightarrow L_{v}^{q}}+\left\|P_{\eta}\right\|_{L_{v}^{q} \rightarrow L_{v}^{q}}\left(\left\|H_{f}^{v}\right\|_{A_{\omega}^{p} \rightarrow L_{v}^{q}}+\left\|H \frac{v}{f}\right\|_{A_{\omega}^{p} \rightarrow L_{v}^{q}}\right) \text {. }
$$

for each $\eta \geq \eta_{0}$. Moreover, there exists $r_{0}=r_{0}(v)>0$ such that for each fixed $r \geq r_{0}$ and $\eta \geq \eta_{0}$

$$
\sup _{z \in \mathbb{D}}\left\|f b_{z, \omega}^{\eta}-\overline{g_{z, \omega}^{\eta}(z)} b_{z, \omega}^{\eta}\right\|_{L_{v}^{q}} \gtrsim \sup _{z \in \mathbb{D}} \gamma(z)\left(\frac{1}{v(\Delta(z, r))} \int_{\Delta(z, r)}\left|f(\zeta)-\overline{g_{z, \omega}^{\eta}(z)}\right|^{q} v(\zeta) d A(\zeta)\right)^{\frac{1}{q}} .
$$

Proof The definition of the Hankel operator along with triangle inequality gives

$$
\begin{aligned}
& \left\|f b_{z, \omega}^{\eta}-\overline{g_{z, \omega}^{\eta}(z)} b_{z, \omega}^{\eta}\right\|_{L_{v}^{q}} \leq\left\|H_{f}^{v}\left(b_{z, \omega}^{\eta}\right)\right\|_{L_{\nu}^{q}}+\left\|P_{v}\left(f b_{z, \omega}^{\eta}\right)-\overline{g_{z, \omega}^{\eta}(z)} b_{z, \omega}^{\eta}\right\|_{L_{v}^{q}} \\
& \leq\left\|H_{f}^{v}\right\|_{A_{\omega}^{p} \rightarrow L_{v}^{q}}\left\|b_{z, \omega}^{\eta}\right\|_{A_{\omega}^{p}}+\left\|P_{v}\left(f b_{z, \omega}^{\eta}\right)-\overline{g_{z, \omega}^{\eta}(z)} b_{z, \omega}^{\eta}\right\|_{L_{v}^{q}} \\
& =\left\|H_{f}^{v}\right\|_{A_{\omega}^{p} \rightarrow L_{v}^{q}}+\left\|P_{\nu}\left(f b_{z, \omega}^{\eta}\right)-\overline{g_{z, \omega}^{\eta}(z)} b_{z, \omega}^{\eta}\right\|_{L_{v}^{q}} \text {. }
\end{aligned}
$$

If $g \in A_{\eta}^{1}$, then the reproducing formula for the standard weighted Bergman projection yields $\overline{g(z)} b_{z, \omega}^{\eta}=P_{\eta}\left(\bar{g} b_{z, \omega}^{\eta}\right)$. Since $v \in B_{q}$ is radial and $f \in L_{v}^{q}$, we have $v \in \mathcal{D}$ and $P_{\nu}\left(f b_{z}^{\eta}\right) \in A_{v}^{q}$ 
by Proposition 6. Therefore $g_{z}^{\eta} \in A_{\nu}^{q}$ for all $z \in \mathbb{D}$. Moreover, $A_{v}^{q} \subset A_{\eta}^{q} \subset A_{\eta}^{1}$ if $\eta>\frac{\beta(v)}{q}-1$ by Lemma A(ii). It follows that

$$
\begin{aligned}
\left\|P_{\nu}\left(f b_{z, \omega}^{\eta}\right)-\overline{g_{z, \omega}^{\eta}(z)} b_{z, \omega}^{\eta}\right\|_{L_{v}^{q}} & =\left\|P_{v}\left(f b_{z, \omega}^{\eta}\right)-P_{\eta}\left(\overline{g_{z, \omega}^{\eta}} b_{z, \omega}^{\eta}\right)\right\|_{L_{v}^{q}} \\
& =\left\|P_{\eta}\left(P_{\nu}\left(f b_{z, \omega}^{\eta}\right)-\overline{g_{z, \omega}^{\eta}} b_{z, \omega}^{\eta}\right)\right\|_{L_{v}^{q}, \quad z \in \mathbb{D} .}
\end{aligned}
$$

By [5], there exists $\eta_{1}=\eta_{1}(v)>\frac{\beta(v)}{q}-1$ such that $P_{\eta}: L_{v}^{q} \rightarrow L_{v}^{q}$ is bounded if $\eta \geq \eta_{1}$. Therefore

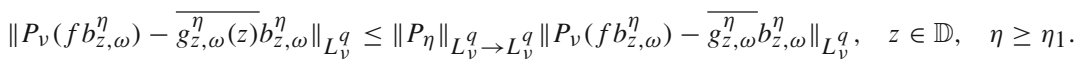

The triangle inequality yields

$$
\begin{aligned}
\left\|P_{v}\left(f b_{z, \omega}^{\eta}\right)-\overline{g_{z, \omega}^{\eta}} b_{z, \omega}^{\eta}\right\|_{L_{v}^{q}} & \leq\left\|f b_{z, \omega}^{\eta}-P_{\nu}\left(f b_{z, \omega}^{\eta}\right)\right\|_{L_{v}^{q}}+\left\|f b_{z, \omega}^{\eta}-\overline{g_{z, \omega}^{\eta}} b_{z, \omega}^{\eta}\right\|_{L_{v}^{q}} \\
& =\left\|H_{f}^{v}\left(b_{z, \omega}^{\eta}\right)\right\|_{L_{v}^{q}}+\left\|\bar{f} b_{z, \omega}^{\eta}-g_{z, \omega}^{\eta} b_{z, \omega}^{\eta}\right\|_{L_{v}^{q}} \\
& \leq\left\|H_{f}^{v}\right\|_{A_{\omega}^{p} \rightarrow L_{v}^{q}}^{q} b_{z, \omega}^{\eta}\left\|_{A_{\omega}^{p}}+\right\| \bar{f} b_{z, \omega}^{\eta}-P_{\nu}\left(\bar{f} b_{z, \omega}^{\eta}\right) \|_{L_{v}^{q}} \\
& =\left\|H_{f}^{v}\right\|_{A_{\omega}^{p} \rightarrow L_{v}^{q}}+\left\|H \frac{v}{f}\left(b_{z, \omega}^{\eta}\right)\right\|_{L_{v}^{q}} \leq\left\|H_{f}^{v}\right\|_{A_{\omega}^{p} \rightarrow L_{v}^{q}}+\left\|H \frac{v}{f}\right\|_{A_{\omega}^{p} \rightarrow L_{v}^{q}} .
\end{aligned}
$$

By combining the above estimates we deduce

$$
\left\|f b_{z, \omega}^{\eta}-\overline{g_{z, \omega}^{\eta}(z)} b_{z, \omega}^{\eta}\right\|_{L_{\nu}^{q}} \leq\left\|H_{f}^{v}\right\|_{A_{\omega}^{p} \rightarrow L_{v}^{q}}+\left\|P_{\eta}\right\|_{L_{v}^{q} \rightarrow L_{\nu}^{q}}\left(\left\|H_{f}^{v}\right\|_{A_{\omega}^{p} \rightarrow L_{v}^{q}}+\left\|H \frac{v}{f}\right\|_{A_{\omega}^{p} \rightarrow L_{v}^{q}}\right),
$$

for any $\eta \geq \eta_{1}(v)$.

To see the second one, first observe that [16, Corollary 2] and Lemma A(ii) give

$$
\begin{aligned}
\left\|B_{z}^{\eta}\right\|_{A_{\omega}^{p}}^{p} & \asymp \int_{0}^{|z|} \frac{\widehat{\omega}(t)}{(1-t)^{p(2+\eta)}} d t \lesssim \frac{\widehat{\omega}(|z|)}{(1-|z|)^{\beta(\omega)}} \int_{0}^{|z|} \frac{1}{(1-t)^{p(2+\eta)-\beta(\omega)}} d t \\
& \asymp \frac{\widehat{\omega}(z)}{(1-|z|)^{p(2+\eta)-1}}, \quad|z| \rightarrow 1^{-},
\end{aligned}
$$

provided $\eta>\frac{\beta(\omega)+1}{p}-2$. Moreover, by (3.6) there exists $r_{0}=r_{0}(v)>0$ such that $(1-$ $|z|) \widehat{v}(z) \asymp v\left(\Delta\left(z, r_{0}\right)\right)$ for any $r \geq r_{0}$. Hence, for each $r \geq r_{0}$ we have

$$
\begin{aligned}
\left\|f b_{z, \omega}^{\eta}-\overline{g_{z, \omega}^{\eta}(z)} b_{z, \omega}^{\eta}\right\|_{L_{v}^{q}}^{q} & \geq\left.\int_{\Delta(z, r)}\left|f(\zeta)-\overline{\left.g_{z, \omega}^{\eta}(z)\right|^{q}}\right| b_{z}^{\eta}(\zeta)\right|^{q} v(\zeta) d A(\zeta) \\
& \asymp \frac{1}{\left\|B_{z}^{\eta}\right\|_{A_{\omega}^{p}(1-|z|)^{q(2+\eta)}}^{q}} \int_{\Delta(z, r)} \mid f(\zeta)-\overline{\left.g_{z, \omega}^{\eta}(z)\right|^{q}} v(\zeta) d A(\zeta) \\
& \asymp \frac{1}{\widehat{\omega}(z)^{\frac{q}{p}}(1-|z|)^{\frac{q}{p}}} \int_{\Delta(z, r)} \mid f(\zeta)-\overline{\left.g_{z, \omega}^{\eta}(z)\right|^{q}} v(\zeta) d A(\zeta), \\
& \asymp \frac{\widehat{v}(z)(1-|z|)}{\widehat{\omega}(z)^{\frac{q}{p}}(1-|z|)^{\frac{q}{p}}} \frac{1}{v(\Delta(z, r))} \int_{\Delta(z, r)} \mid f(\zeta)-\overline{\left.g_{z, \omega}^{\eta}(z)\right|^{q}} v(\zeta) d A(\zeta) .
\end{aligned}
$$

The second claim for $\eta_{0}=\max \left\{\eta_{1}, \frac{\beta(\omega)+1}{p}-2\right\}$ is now proved.

Proof of Theorem 1 If $H_{f}^{v}, H_{f}^{v}: A_{\omega}^{p} \rightarrow L_{v}^{q}$ are bounded, then $f \in \mathrm{BMO}(\Delta)$ by Proposition 15 and Theorem 11.

Conversely, let $f \in \operatorname{BMO}(\Delta)$. Then $f$ can be decomposed as $f=f_{1}+f_{2}$, where $f_{1} \in \mathrm{BA}(\Delta)$ and $f_{2} \in \mathrm{BO}(\Delta)$, by Theorem 11(ii). Proposition 14 shows that $H_{f_{2}}^{v}, H \frac{v}{f_{2}}$ : 
$A_{\omega}^{p} \rightarrow L_{v}^{q}$ are bounded. Moreover, since $v \in B_{q}$ is radial, $v \in \mathcal{D}$ and $P_{v}: L_{v}^{q} \rightarrow L_{v}^{q}$ is bounded by Proposition 6 . Therefore Lemma 10 yields

$$
\left\|H_{f_{1}}^{v}(g)\right\|_{L_{v}^{q}}^{q} \leq\left\|f_{1} g\right\|_{L_{v}^{q}}+\left\|P_{v}\left(f_{1} g\right)\right\|_{L_{v}^{q}} \lesssim\left\|f_{1} g\right\|_{L_{v}^{q}} \lesssim\|g\|_{A_{\omega}^{p}} g \in H^{\infty} .
$$

It follows that $H_{f}^{v}, H_{\frac{v}{f}}^{v}: A_{\omega}^{p} \rightarrow L_{v}^{q}$ are bounded.

\section{Anti-analytic symbols}

Recall that the space $\mathcal{B}_{d \gamma}$ consists of $f \in \mathcal{H}(\mathbb{D})$ such that

$$
\|f\|_{\mathcal{B}_{d \gamma}}=\sup _{z \in \mathbb{D}}\left|f^{\prime}(z)\right|(1-|z|) \gamma(z)+|f(0)|<\infty,
$$

where $\gamma(z)=\frac{\widehat{v}(z)^{\frac{1}{q}}(1-|z|)^{\frac{1}{q}}}{\widehat{\omega}(z)^{\frac{1}{p}}(1-|z|)^{\frac{1}{p}}}$ for all $z \in \mathbb{D}$.

Proposition 16 Let $1<p \leq q<\infty, \omega, v \in \mathcal{D}$ and $r \geq r_{0}$, where $r_{0}=r_{0}(v)>0$ is that of Theorem 11(i). Then $\operatorname{BMO}(\Delta) \cap \mathcal{H}(\mathbb{D})=\operatorname{BMO}(\Delta)_{\omega, v, p, q, r} \cap \mathcal{H}(\mathbb{D})=\mathcal{B}_{d \gamma}$.

Proof Let first $f \in \mathcal{B}_{d \gamma}$. By Theorem 11(iv) to deduce $f \in \operatorname{BMO}(\Delta)$ it is enough to prove

$$
\sup _{z \in \mathbb{D}} \frac{(1-|z|)^{c+1} \gamma(z)^{q}}{\widehat{v}(z)} \int_{\mathbb{D}}|f(\zeta)-f(z)|^{q} \frac{\left(1-|\zeta|^{2}\right)^{\sigma}}{|1-z \bar{\zeta}|^{2+c+\sigma}} v(\zeta) d A(\zeta)<\infty
$$

for some $\sigma>0$ and

$$
c>2 \frac{q}{p}(\beta(\omega)+1)+\sigma+\max \{2 \beta(\nu), \gamma(v)\} .
$$

Since $f \in \mathcal{H}(\mathbb{D})$, the function $(f(\zeta)-f(z))(1-\zeta \bar{z})^{-\frac{2+c+\sigma}{q}}$ is an analytic function in $\zeta$ for each $z \in \mathbb{D}$. Therefore Lemma 5 shows that (6.1) is equivalent to

$$
\sup _{z \in \mathbb{D}} \frac{(1-|z|)^{c+1} \gamma(z)^{q}}{\widehat{v}(z)} \int_{\mathbb{D}}|f(\zeta)-f(z)|^{q} \frac{\left(1-|\zeta|^{2}\right)^{\sigma-1}}{|1-z \bar{\zeta}|^{2+c+\sigma}} \widehat{v}(\zeta) d A(\zeta)<\infty .
$$

Further, Lemma A(ii) yields

$$
\begin{aligned}
& \frac{(1-|z|)^{c+1} \gamma(z)^{q}}{\widehat{v}(z)} \int_{\mathbb{D}}|f(\zeta)-f(z)|^{q} \frac{\left(1-|\zeta|^{2}\right)^{\sigma-1}}{|1-z \bar{\zeta}|^{2+c+\sigma}} \widehat{v}(\zeta) d A(\zeta) \\
& \lesssim(1-|z|)^{c+1} \gamma(z)^{q} \int_{\mathbb{D} \backslash D(0,|z|)}|f(\zeta)-f(z)|^{q} \frac{\left(1-|\zeta|^{2}\right)^{\sigma-1}}{|1-z \bar{\zeta}|^{2+c+\sigma}} d A(\zeta) \\
& \quad+(1-|z|)^{c+1-\beta(v)} \gamma(z)^{q} \int_{D(0,|z|)}|f(\zeta)-f(z)|^{q} \frac{\left(1-|\zeta|^{2}\right)^{\sigma+\beta(v)-1}}{|1-z \bar{\zeta}|^{2+c+\sigma}} d A(\zeta) \\
& \leq(1-|z|)^{c+1} \gamma(z)^{q} \int_{\mathbb{D}}|f(\zeta)-f(z)|^{q} \frac{\left(1-|\zeta|^{2}\right)^{\sigma-1}}{|1-z \bar{\zeta}|^{2+c+\sigma}} d A(\zeta) \\
& \quad+(1-|z|)^{c+1-\beta(v)} \gamma(z)^{q} \int_{\mathbb{D}}|f(\zeta)-f(z)|^{q} \frac{\left(1-|\zeta|^{2}\right)^{\sigma+\beta(v)-1}}{|1-z \bar{\zeta}|^{2+c+\sigma}} d A(\zeta) \\
& =I_{1}(z)+I_{2}(z), \quad z \in \mathbb{D} .
\end{aligned}
$$


Fix $\sigma>\max \left\{0,1-\frac{q}{p}(1+\alpha(\omega))+q \beta(v)\right\}$ and $c$ satisfying (6.2). Then

$$
c>\max \left\{\beta(v)-1,-2+\beta(v)+\frac{q}{p}(1+\beta(\omega))-q \alpha(v)\right\} .
$$

Therefore, [11, Lemma 7] together with Lemmas A(ii) and B gives

$$
\begin{aligned}
I_{1}(z) & \lesssim(1-|z|)^{c+1} \gamma(z)^{q} \int_{\mathbb{D}}\left|f^{\prime}(\zeta)\right|^{q} \frac{\left(1-|\zeta|^{2}\right)^{\sigma+q-1}}{|1-z \bar{\zeta}|^{2+c+\sigma}} d A(\zeta) \\
& \lesssim\|f\|_{\mathcal{B}_{d \gamma}}^{q}(1-|z|)^{c+1} \gamma(z)^{q} \int_{\mathbb{D}} \gamma(\zeta)^{-q} \frac{\left(1-|\zeta|^{2}\right)^{\sigma-1}}{|1-z \bar{\zeta}|^{2+c+\sigma}} d A(\zeta) \\
& \asymp\|f\|_{\mathcal{B}_{d \gamma}}^{q}(1-|z|)^{c+1} \gamma(z)^{q} \int_{0}^{1} \frac{\widehat{\omega}(s)^{\frac{q}{p}}}{\widehat{\widehat{v}(s)}} \frac{(1-s)^{\frac{q}{p}+\sigma-2}}{(1-s|z|)^{1+\sigma+c}} d s \\
& \lesssim\|f\|_{\mathcal{B}_{d \gamma}}^{q}(1-|z|)^{c+1} \gamma(z)^{q} \frac{\widehat{\omega}(z)^{\frac{q}{p}}(1-|z|)^{\alpha(v)}}{(1-|z|)^{\frac{q}{p} \beta(\omega)} \widehat{v}(z)} \int_{0}^{|z|} \frac{(1-s)^{3+c-\frac{q}{p}-\frac{q}{p} \beta(\omega)+\alpha(v)}}{(1-|z|)^{\beta(v)}} \int_{|z|}^{1}(1-s)^{\frac{q}{p}+\sigma-2+\frac{q}{p} \alpha(\omega)-\beta(v)} d s \\
& +\|f\|_{\mathcal{B}_{d \gamma}}^{q}(1-|z|)^{-\sigma} \gamma(z)^{q} \frac{\widehat{\omega}(z)^{\frac{q}{p}}(1-|z|)^{\frac{q}{p} \alpha(\omega)} \widehat{v}(z)}{(1-\mid z)} \\
& \lesssim\|f\|_{\mathcal{B}_{d \gamma}}^{q}(1-|z|)^{-1+\frac{q}{p}} \gamma(z)^{q} \frac{\widehat{\omega}(z)^{\frac{q}{p}}}{\widehat{v}(z)} \asymp\|f\|_{\mathcal{B}_{d \gamma}}^{q}<\infty, \quad z \in \mathbb{D},
\end{aligned}
$$

and

$$
\begin{aligned}
I_{2}(z) & \lesssim(1-|z|)^{c+1-\beta(v)} \gamma(z)^{q} \int_{\mathbb{D}}\left|f^{\prime}(\zeta)\right|^{q} \frac{\left(1-|\zeta|^{2}\right)^{\sigma+\beta(v)+q-1}}{|1-z \bar{\zeta}|^{2+c+\sigma}} d A(\zeta) \\
& \lesssim\|f\|_{\mathcal{B}_{d \gamma}}^{q}(1-|z|)^{c+1-\beta(v)} \gamma(z)^{q} \int_{\mathbb{D}} \gamma(\zeta)^{-q} \frac{\left(1-|\zeta|^{2}\right)^{\beta(v)+\sigma-1}}{|1-z \bar{\zeta}|^{2+c+\sigma}} d A(\zeta) \\
& \asymp\|f\|_{\mathcal{B}_{d \gamma}}^{q}(1-|z|)^{c+1-\beta(v)} \gamma(z)^{q} \int_{0}^{1} \frac{\widehat{\omega}(s)^{\frac{q}{p}}}{\widehat{\widehat{v}(s)}} \frac{(1-s)^{\beta(v)+\frac{q}{p}+\sigma-2}}{(1-s|z|)^{1+\sigma+c}} d s \\
& \lesssim\|f\|_{\mathcal{B}_{d \gamma}}^{q}(1-|z|)^{c+1-\beta(v)} \gamma(z)^{q} \frac{\widehat{\omega}(z)^{\frac{q}{p}}(1-|z|)^{\alpha(v)}}{(1-|z|)^{\frac{q}{p} \beta(\omega)} \widehat{v}(z)^{q}} \int_{0}^{|z|} \frac{d s}{(1-s)^{3+c-\beta(v)-\frac{q}{p}-\frac{q}{p} \beta(\omega)+\alpha(v)}} \\
& +\|f\|_{\mathcal{B}_{d \gamma}}^{q}(1-|z|)^{-\sigma-\beta(v)} \gamma(z)^{q} \frac{\widehat{\omega}(z)^{\frac{q}{p}}(1-|z|)^{\beta(v)}}{(1-|z|)^{\frac{q}{p} \alpha(\omega)} \widehat{v}(z)} \int_{|z|}^{1}(1-s)^{\beta(v)+\frac{q}{p}+\sigma-2+\frac{q}{p} \alpha(\omega)-\beta(v)} d s \\
& \lesssim\|f\|_{\mathcal{B}_{d \gamma}}^{q}(1-|z|)^{-1+\frac{q}{p}} \gamma(z)^{q} \frac{\widehat{\omega}(z)^{\frac{q}{p}}}{\widehat{v}(z)^{q}} \asymp\|f\|_{\mathcal{B}_{d \gamma}}^{q}<\infty, \quad z \in \mathbb{D} .
\end{aligned}
$$

By combining these estimates we deduce $f \in \operatorname{BMO}(\Delta)$, and thus $\mathcal{B}_{d \gamma} \subset \mathcal{H}(\mathbb{D}) \cap \operatorname{BMO}(\Delta)$.

Assume now that $f \in \mathcal{H}(\mathbb{D}) \cap \operatorname{BMO}(\Delta)$. Then (6.3) holds for some $\sigma>1$ and $c$ satisfying (6.2). Therefore (3.6) implies

$$
\begin{aligned}
\infty & >\sup \frac{(1-|z|)^{c+1} \gamma(z)^{q}}{\widehat{v}(z)} \int_{\mathbb{D}}|f(\zeta)-f(z)|^{q} \frac{\left(1-|\zeta|^{2}\right)^{\sigma-1}}{|1-z \bar{\zeta}|^{2+c+\sigma}} \widehat{v}(\zeta) d A(\zeta) \\
& \gtrsim \frac{\gamma(z)^{q}}{(1-|z|)^{2} \widehat{v}(z)} \int_{\Delta(z, r)}|f(\zeta)-f(z)|^{q} \widehat{v}(\zeta) d A(\zeta) \\
& \asymp \frac{\gamma(z)^{q}}{|\Delta(z, r)|} \int_{\Delta(z, r)}|f(\zeta)-f(z)|^{q} d A(\zeta), \quad z \in \mathbb{D} .
\end{aligned}
$$


By arguing as in $[12,1653-1654]$ we deduce $\mathcal{H}(\mathbb{D}) \cap \operatorname{BMO}(\Delta) \subset \mathcal{B}_{d \gamma}$.

The space $\mathcal{B}_{d \gamma}$ consists of constant functions only if $\lim \sup _{|z| \rightarrow 1^{-}}((1-|z|) \gamma(|z|))^{-1}=0$. Moreover, $\mathcal{B}_{d \gamma}$ is a subset of the disc algebra if $((1-x) \gamma(x))^{-1} \in L^{1}(0,1)$, and $\mathcal{B}_{d \gamma}$ coincides with a Bloch-type space if $\gamma$ is decreasing.

Proof of Theorem 2 Since $f \in A_{v}^{1}$, the operator $H_{\frac{v}{f}}$ is densely defined. If $H_{\frac{v}{f}}: A_{\omega}^{p} \rightarrow L_{v}^{q}$ is bounded, choosing $g \equiv 1$ it follows that $f \in A_{\nu}^{q}$, and therefore $f \in \mathcal{B}_{d \gamma}$ by Theorem 1 and Proposition 16.

Conversely, assume $f \in \mathcal{B}_{d \gamma}$. Since $v \in B_{q}$ is radial, Proposition 6 implies $v \in \mathcal{D}$. Therefore Lemmas A(ii) and B yield

$$
\begin{aligned}
\|f\|_{A_{v}^{q}}^{q} & \lesssim \int_{\mathbb{D}}\left(\int_{0}^{|z|}\left|f^{\prime}\left(s \frac{z}{|z|}\right)\right| d s\right)^{q} v(z) d A(z)+|f(0)|^{q} \\
& \lesssim\|f\|_{\mathcal{B}_{d \gamma}}^{q}\left(1+\int_{0}^{1}\left(\int_{0}^{t} \frac{d s}{(1-s) \gamma(s)}\right)^{q} v(t) d t\right) \\
& \lesssim\|f\|_{\mathcal{B}_{d \gamma}}^{q}\left(1+\int_{0}^{1}\left(\int_{0}^{t} \frac{\widehat{\omega}(s)^{\frac{1}{p}}}{\widehat{v}(s)^{\frac{1}{q}}(1-s)^{1+\frac{1}{q}-\frac{1}{p}}} d s\right)^{q} v(t) d t\right) \\
& \lesssim\|f\|_{\mathcal{B}_{d \gamma}}^{q}\left(1+\int_{0}^{1} \frac{\widehat{\omega}(t)^{\frac{q}{p}}}{\widehat{v}(t)(1-t)^{\frac{q \alpha(\omega)}{p}-\beta(v)}}\left(\int_{0}^{t} \frac{d s}{(1-s)^{1+\frac{1+\beta(v)}{q}-\frac{1+\alpha(\omega)}{p}}}\right)^{q} v(t) d t\right)
\end{aligned}
$$

for all $f \in \mathcal{H}(\mathbb{D})$. If $\frac{1+\beta(v)}{q}-\frac{1+\alpha(\omega)}{p}>0$, Lemma 3(ii) gives

$$
\begin{aligned}
\|f\|_{A_{v}^{q}}^{q} & \lesssim\|f\|_{\mathcal{B}_{d \gamma}}^{q}\left(1+\int_{0}^{1} \frac{\widehat{\omega}(t)^{\frac{q}{p}}(1-t)^{\frac{q}{p}-1}}{\widehat{v}(t)} v(t) d t\right) \\
& \lesssim\|f\|_{\mathcal{B}_{d \gamma}}^{q}\left(1+\widehat{\omega}(0)^{\frac{q}{p}-1} \int_{0}^{1} \frac{\widehat{\omega}(t) v(t)}{\widehat{v}(t)} d t\right) \lesssim\|f\|_{\mathcal{B}_{d \gamma}}^{q} .
\end{aligned}
$$

If $\frac{1+\beta(v)}{q}-\frac{1+\alpha(\omega)}{p}=0$, then Lemmas B and 3(ii) yield

$$
\begin{aligned}
\|f\|_{A_{v}^{q}}^{q} & \lesssim\|f\|_{\mathcal{B}_{d \gamma}}^{q}\left(1+\int_{0}^{1} \frac{\widehat{\omega}(t)^{\frac{q}{p}}(1-t)^{\frac{q}{p}-1}}{\widehat{v}(t)} \log \frac{e}{1-t} v(t) d t\right) \\
& \lesssim\|f\|_{\mathcal{B}_{d \gamma}}^{q} \widehat{\omega}(0)^{\frac{q}{p}} \int_{0}^{1} \frac{(1-t)^{\alpha(\omega) \frac{q}{p}+\frac{q}{p}-1}}{\widehat{v}(t)} \log \frac{e}{1-t} v(t) d t \\
& \lesssim\|f\|_{\mathcal{B}_{d \gamma}}^{q} \int_{0}^{1} \frac{(1-t)^{\alpha(\omega) \frac{q}{2 p}+\frac{q}{p}-1}}{\widehat{v}(t)} v(t) d t \lesssim\|f\|_{\mathcal{B}_{d \gamma}}^{q} .
\end{aligned}
$$

Finally, if $\frac{1+\beta(v)}{q}-\frac{1+\alpha(\omega)}{p}<0$, then Lemma 3(ii) gives

$$
\|f\|_{A_{v}^{q}}^{q} \lesssim\|f\|_{\mathcal{B}_{d \gamma}}^{q}\left(1+\int_{0}^{1} \frac{\widehat{\omega}(t)^{\frac{q}{p}}(1-t)^{\frac{q}{p}-1}}{\widehat{v}(t)} v(t) d t\right) \lesssim\|f\|_{\mathcal{B}_{d \gamma}}^{q} .
$$

Therefore $f \in A_{v}^{q}$, and thus $\mathcal{B}_{d \gamma} \subset A_{v}^{q}$. This together with Theorem 1 and Proposition 16 finishes the proof. 
Acknowledgements Open access funding provided by University of Gothenburg.

Open Access This article is distributed under the terms of the Creative Commons Attribution 4.0 International License (http://creativecommons.org/licenses/by/4.0/), which permits unrestricted use, distribution, and reproduction in any medium, provided you give appropriate credit to the original author(s) and the source, provide a link to the Creative Commons license, and indicate if changes were made.

\section{References}

1. Arazy, J., Fisher, S.D., Peetre, J.: Hankel operators on weighted Bergman spaces. Am. J. Math. 110(6), 989-1053 (1988)

2. Arrousi, H.: Function and Operator Theory on Large Bergman Spaces. PhD. Thesis, Univ. of Barcelona (2016)

3. Axler, S.: The Bergman space, the Bloch space, and commutators of multiplication operators. Duke Math. J. 53(2), 315-332 (1986)

4. Bekollé, D.: Inégalités á poids pour le projecteur de Bergman dans la boule unité de $C^{n}$ [Weighted inequalities for the Bergman projection in the unit ball of $C^{n}$ ]. Stud. Math. 71(3), 305-323 (1981/82)

5. Bekollé, D., Bonami, A.: Inégalités á poids pour le noyau de Bergman (French). C. R. Acad. Sci. Paris Sér. A-B 286(18), 775-778 (1978)

6. Bekollé, D., Berger, C., Coburn, L.A., Zhu, K.: BMO in the Bergman metric on bounded symmetric domains. J. Funct. Anal. 93(2), 310-350 (1990)

7. Berger, C., Coburn, L .A., Zhu, K.: BMO on the Bergman spaces of the classical domains. Bull. Am. Math. Soc. (N.S.) 17(1), 133-136 (1987)

8. Berger, C., Coburn, L.A., Zhu, K.: Function theory on Cartan domains and the Berezin-Toeplitz symbol calculus. Am. J. Math. 110(5), 921-953 (1988)

9. Constantin, O., Peláez, J.A.: Boundedness of the Bergman projection on $L^{p}$-spaces with exponential weights. Bull. Sci. Math. 139, 245-268 (2015)

10. Galanopoulos, P., Pau, J.: Hankel operators on large weighted Bergman spaces. Ann. Acad. Sci. Fenn. Math. 37(2), 635-648 (2012)

11. Pau, J., Zhao, R.: Weak factorization and Hankel forms for weighted Bergman spaces on the unit ball. Math. Ann. 363(1-2), 363-383 (2015)

12. Pau, J., Zhao, R., Zhu, K.: Weighted BMO and Hankel operators between Bergman spaces. Indiana Univ. Math. J. 65(5), 1639-1673 (2016)

13. Peláez, J.A.: Small weighted Bergman spaces. In: Proceedings of the Summer School in Complex and Harmonic Analysis, and Related Topics (2016)

14. Peláez, J.A., Rättyä, J.: Weighted Bergman Spaces Induced by Rapidly Increasing Weights, vol. 227. Mem. Am. Math. Soc. (2014)

15. Peláez, J.A., Rättyä, J.: Embedding theorems for Bergman spaces via harmonic analysis. Math. Ann. 362(1-2), 205-239 (2015)

16. Peláez, J.A., Rättyä, J.: Two weight inequality for Bergman projection. J. Math. Pures Appl. 105, 102-130 (2016)

17. Peláez, J.A., Sierra, K., Rättyä, J.: Atomic decomposition and Carleson measures for weighted Mixed norm spaces. arXiv:1709.07239

18. Perälä, A.: Vanishing Bergman kernels on the disk. J. Geom. Anal. 28(2), 1716-1727 (2018)

19. Zhao, R.: Generalization of Schur's test and its application to a class of integral operators on the unit ball of $\mathbb{C}^{n}$. Integral Equ. Oper. Theory 82(4), 519-532 (2015)

20. Zhao, R., Zhu, K.: Theory of Bergman spaces in the unit ball of $\mathbb{C}^{n}$. Mem. Soc. Math. Fr. (N.S.) no. 115 (2008), p. vi+103 (2009)

21. Zhu, K.: VMO, ESV, and Toeplitz operators on the Bergman space. Trans. Am. Math. Soc. 302(2), 617-646 (1987)

22. Zhu, K.: BMO and Hankel operators on Bergman spaces. Pac. J. Math. 155(2), 377-395 (1992)

23. Zhu, K.: Operator Theory in Function Spaces, Math. Surveys and Monographs, vol. 138, 2nd edn. American Mathematical Society, Providence (2007)

Publisher's Note Springer Nature remains neutral with regard to jurisdictional claims in published maps and institutional affiliations. 